\title{
Capillary Waves on the Surface of Simple Liquids Measured by X-Ray Reflectivity
}

\section{Citation}

Braslau, A., Peter S. Pershan, G. Swislow, B. M. Ocko, and J. Als-Nielsen. 1988. Capillary waves on the surface of simple liquids measured by $x$-ray reflectivity. Physical Review A 38(5): 2457-2470.

\section{Published Version}

doi:10.1103/PhysRevA.38.2457

\section{Permanent link}

http://nrs.harvard.edu/urn-3:HUL.InstRepos:10357472

\section{Terms of Use}

This article was downloaded from Harvard University's DASH repository, and is made available under the terms and conditions applicable to Other Posted Material, as set forth at http:// nrs.harvard.edu/urn-3:HUL.InstRepos:dash.current.terms-of-use\#LAA

\section{Share Your Story}

The Harvard community has made this article openly available.

Please share how this access benefits you. Submit a story.

\section{Accessibility}




\title{
Capillary waves on the surface of simple liquids measured by $x$-ray reflectivity
}

\author{
A. Braslau, P. S. Pershan, and G. Swislow \\ Department of Physics and Division of Applied Sciences, Harvard University, Cambridge, Massachusetts 02138 \\ B. M. Ocko \\ Department of Physics, Brookhaven National Laboratory, Upton, New York 11973 \\ and Department of Physics and Division of Applied Sciences, Harvard University, Cambridge, Massachusetts 02138 \\ J. Als-Nielsen \\ Risø National Laboratory, DK-4000 Roskilde, Denmark
}

(Received 25 January 1988)

\begin{abstract}
The properties of the liquid-vapor interface for three simple liquids (water, carbon tetrachloride, and methanol) have been measured using $x$-ray reflectivity. The measured surface roughness is interpreted using a model that combines the effects of thermally induced capillary waves and the dimensions of the constituent molecules.
\end{abstract}

\section{INTRODUCTION}

The structural properties of liquid surfaces are of great interest, but techniques commonly used for the study of solid surfaces (scattering, electron tunneling, and microscopy) are technically difficult or even impossible to apply to liquids. Recently, however, the technique of x-ray reflectivity has been applied to the study of liquid surfaces with considerable success. ${ }^{1-7}$ The reflectivity technique is sensitive not only to the local surface density profile, but also to height fluctuations from thermally induced surface capillary waves.

This paper presents a detailed theoretical treatment of the $\mathrm{x}$-ray reflectivity technique, with particular emphasis on its application to capillary waves. The measured x-ray reflectivity from a liquid surface is modeled by the Fresnel reflectivity for a perfectly flat step interface multiplied by a Gaussian factor of the form $\exp \left(-Q_{z}^{2} \sigma_{T}^{2}\right)$, where $\sigma_{T}^{2}=\sigma_{P}^{2}+\sigma_{C}^{2}\left(Q_{z}\right)$. The first width, denoted $\sigma_{P}$, corresponds to the local surface profile. The other, $\sigma_{C}\left(Q_{z}\right)$, is the effective capillary-wave surface roughness averaged over the spectrometer coherence area. The data are fit to the single parameter $\sigma_{P}$, whereas $\sigma_{C}$ is calculated exactly.

For the water surface, $\sigma_{P}$ is in good agreement with the molecular size of water as previously reported, ${ }^{3}$ and reaffirms the continuum capillary-wave model. ${ }^{8,9}$ The essential elements of the model were presented with that result. We also report $x$-ray reflectivity measurements on two other simple liquids, methanol and carbon tetrachloride. For carbon tetrachloride, the fitted surfaceprofile width is also in reasonable agreement with the molecular size. In addition, the predicted effect on $\sigma_{C}$ of the $x$-ray spectrometer resolution function on the measured reflectivity is confirmed. The fitted surface-profile width of methanol is significantly smaller than the molecular size. Interpretations of this discrepancy, discussed in the experimental results section, include the possibility the effective surface tension for short-wavelength capillary waves may be larger than the nominal value. Theoretical expressions for the nonspecular diffuse scattering by surface roughness are presented in the Appendix.

\section{THEORY OF X-RAY REFLECTIVITY}

\section{A. Fresnel equation}

The index of refraction for $\mathrm{x}$ rays in matter is less than unity, and thus they are totally externally reflected from a surface at sufficiently low angles of incidence. For a perfectly flat surface with a sharp interface to the vapor, $x$ rays obey the Fresnel law of optics. However, for a diffuse or rough interface, the reflectivity falls off faster than the Fresnel law, with the difference being a measure of the characteristic length of the electron-density termination. ${ }^{1-4}$ The Fresnel law of specular reflection is similar to the Darwin theory for the line shape associated with Bragg reflection from a sharply terminated crystal lattice. ${ }^{10,11}$ If the crystal lattice terminates smoothly, the tails of the scattering fall off more rapidly than the Darwin theory. Measurements of both the specular and Bragg reflectivity allow determination of the characteristic termination lengths of both the total electron density and the electron density associated with the crystal lattice.

The reflectivity of $\mathrm{x}$ rays from a flat, ideal surface can be calculated from the standard expressions of classical optics by substituting the following expressions for the real and imaginary parts of the dielectric constant,

$$
\epsilon^{\prime}=1-\left(\frac{\rho_{e} \lambda^{2}}{\pi}\right)\left(\frac{e^{2}}{m c^{2}}\right), \quad \epsilon^{\prime \prime}=\frac{\beta \lambda}{2 \pi},
$$

where $\rho_{e}$ is the electron density, $\lambda$ is the $\mathrm{x}$-ray wave- 
length, and $\beta^{-1}$ is the absorption length. For simplicity we will always use the small-angle approximation $[\sin (\theta) \approx \theta$ ] where the angle of incidence $\theta$ is measured from the plane of the surface. With this approximation, the Fresnel equations for the reflectivity ${ }^{12}$ reduce to

$$
R_{F}(\theta)=\left|\frac{\theta-\left(\theta^{2}-\theta_{c}^{2}-i \epsilon^{\prime \prime}\right)^{1 / 2}}{\theta+\left(\theta^{2}-\theta_{c}^{2}+i \epsilon^{\prime \prime}\right)^{1 / 2}}\right|^{2},
$$

where $\theta_{c}=\left(1-\epsilon^{\prime}\right)^{1 / 2}=\left(\rho_{e} e^{2} \lambda^{2} / \pi m c^{2}\right)^{1 / 2}$ is the critical angle for total external reflection. The critical wave vector, $Q_{c}=(4 \pi / \lambda) \theta_{c}$ is independent of wavelength. Numerically, $Q_{c}=\left(3.75 \times 10^{-14}\right) \rho_{e}^{1 / 2} \AA^{-1}$, where $\rho_{e}$ is in electrons $/ \mathrm{cm}^{3}$.

If absorption is neglected, the Fresnel law can be written as

$$
\begin{aligned}
R_{F}(\theta) & =\left[\frac{\theta-\left(\theta^{2}-\theta_{c}^{2}\right)^{1 / 2}}{\theta+\left(\theta^{2}-\theta_{c}^{2}\right)^{1 / 2}}\right]^{2} \\
& =\left\lfloor\frac{\theta_{c}}{\theta+\left(\theta^{2}-\theta_{c}^{2}\right)^{1 / 2}}\right]^{4} .
\end{aligned}
$$

For $\theta \gg \theta_{c}$ Eq. (3) reduces to $R_{F}(\theta) \approx\left(\theta_{c} / 2 \theta\right)^{4}$. This approximation and Eq. (3) are plotted in Fig. 1 for $\theta / \theta_{c} \lesssim 15$. For $\theta / \theta_{c} \gtrsim 5$ the two forms agree to within $4 \%$.

The effect of absorption $\left(\epsilon^{\prime \prime}>0\right)$ is only important close to the critical angle, as demonstrated in Fig. 2, which is a plot of Eq. (2) for various values of the absorption coefficient. Table I lists values of the absorption lengths and other parameters for the materials studied.

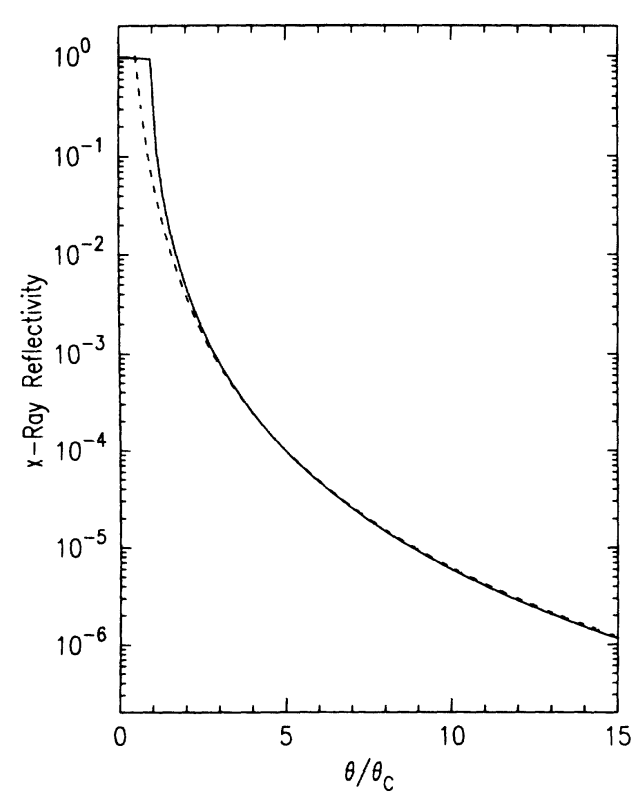

FIG. 1. Calculated $x$-ray reflectivity at small angles of incidence from an ideal surface of water. The $\mathrm{x}$-ray wavelength is $\lambda=1.54 \AA$. The solid line is the Fresnel law of classical optics and the dashed line is the approximation for $\theta \gg \theta_{c}$ : $R_{F}(\theta) \approx\left(\theta_{c} / 2 \theta\right)^{4} ; \theta_{c}=0.0217 \AA^{-1}$.

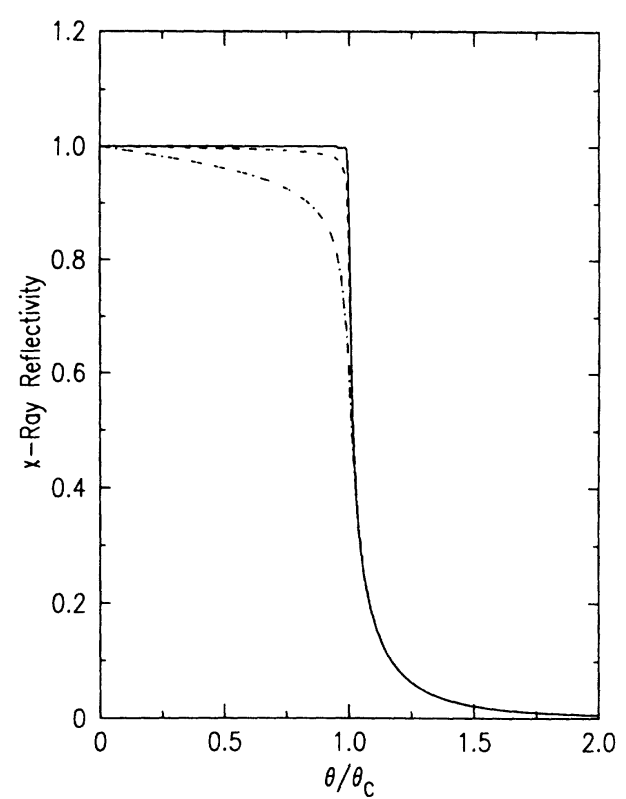

FIG. 2. Effect of absorption on the Fresnel reflectivity near the critical angle. The solid line neglects absorption, the shortdashed line is for an absorption length of $1 \mathrm{~mm}$ (water), and the dot-dashed line is for an absorption length of $0.1 \mathrm{~mm}$ (carbon tetrachloride).

The reflectivity from a real surface departs from the Fresnel law $R_{F}(\theta)$ when the surface is not ideally flat or the transition to the vapor is not ideally sharp. If scattering is at angles sufficiently greater than the critical angle, the expected reflectivity from nonideal surfaces can be calculated using the classical equivalent of the "first Born approximation." 13

\section{B. Born approximation}

In this approximation, multiple scattering of $x$ rays is neglected. The differential scattering cross section is given by

$$
\frac{d \sigma}{d \Omega}=\frac{\left|\hat{\boldsymbol{\epsilon}}^{*} \mathbf{A}_{\mathrm{sc}}\right|^{2}}{\left|\mathbf{D}_{0}\right|^{2}},
$$

where $\hat{\boldsymbol{\epsilon}}^{*}$ is the unit vector along the polarization direction of the scattered radiation and

TABLE I. Absorption coefficient and other parameters for materials studied. Listed are the electron density $\rho_{e}$, the mass density $\rho$, the critical wave vector $Q_{c}$, and the absorption length $\beta^{-1}$ at $\lambda=1.54 \AA$.

\begin{tabular}{ccccl}
\hline \hline Sample & $\begin{array}{c}\rho_{e} \\
\left(\text { electrons } / \mathrm{cm}^{3}\right)\end{array}$ & $\begin{array}{c}\rho \\
\left(\mathrm{g} / \mathrm{cm}^{3}\right)\end{array}$ & $\begin{array}{c}Q_{c} \\
\left(\AA^{-1}\right)\end{array}$ & $\begin{array}{l}\beta^{-1} \\
(\mathrm{~cm})\end{array}$ \\
\hline $\mathrm{H}_{2} \mathrm{O}$ & $3.34 \times 10^{23}$ & 1.000 & 0.0217 & 0.10 \\
$\mathrm{CCl}_{4}$ & $4.60 \times 10^{23}$ & 0.7914 & 0.0254 & 0.010 \\
$\mathrm{CH}_{3} \mathrm{OH}$ & $2.68 \times 10^{23}$ & 1.587 & 0.0194 & 0.14 \\
\hline \hline
\end{tabular}




$$
\mathbf{A}_{\mathrm{sc}}=\frac{1}{4 \pi} \int d^{3} \mathbf{r} e^{-i \mathbf{k} \cdot \mathrm{r}}[\nabla \times(\nabla \times 4 \pi \mathbf{P})] .
$$

If the polarization density in the material is

$$
\mathbf{P}=-\left(e^{2} / m \omega^{2}\right) \rho_{e}(\mathbf{r}) \mathbf{E}_{0}(\mathbf{r}),
$$

where $\rho_{e}(\mathbf{r})$ is the local electron density, $\omega$ is the $\mathrm{x}$-ray frequency, and $\mathbf{E}_{0}(\mathbf{r})=\mathbf{D}_{0} \exp \left(-i \mathbf{k}_{0} \cdot \mathbf{r}\right)$ is the incident field when absorption and refraction are neglected, then

$$
\begin{aligned}
\frac{\hat{\boldsymbol{\epsilon}}^{*} \mathbf{A}_{\mathrm{sc}}}{\left|\mathbf{D}_{0}\right|}=\left[\frac{e^{2}}{m \omega^{2}}\right] k^{2} \int & d^{3} \mathbf{r} e^{-i\left(\mathbf{k}-\mathbf{k}_{0}\right) \cdot \mathbf{r}} \\
& \times \hat{\boldsymbol{\epsilon}}^{*} \cdot\left[\hat{\mathbf{n}} \times\left(\hat{\mathbf{n}} \times \hat{\boldsymbol{\epsilon}}_{0}\right)\right] \rho_{e}(\mathbf{r}),
\end{aligned}
$$

where $\hat{\mathbf{n}}$ and $\hat{\boldsymbol{\epsilon}}_{0}$ are, respectively, the unit vectors along the direction of the incident wave vector and the incident $E$ field. The wave vectors $\mathbf{k}_{0}$ and $\mathbf{k}$ correspond to the incident and scattered fields. For small angles of incidence, polarization effects can also be neglected to obtain

$$
\frac{d \sigma}{d \Omega}=\left(\frac{\theta_{c}^{2} k^{2}}{4 \pi \rho_{e}}\right)^{2}\left|\int d^{3} \mathbf{r} e^{-i \mathbf{Q} \cdot \mathbf{r}} \rho_{e}(\mathbf{r})\right|^{2},
$$

where $\mathbf{Q}=\mathbf{k}-\mathbf{k}_{0}$.

Figure 3(a) illustrates the coordinate system to be used. The surface lies in the $x-y$ plane, and the direction of $\widehat{z}$ is from the material into the vacuum or vapor phase. The incident wave vector $\mathbf{k}_{0}$ lies in the $y-z$ plane.

For a perfectly flat surface, the integrals over $x$ and $y$ yield $4 \pi^{2} \delta^{(2)}\left(Q_{x y}\right) A_{0} / \theta_{0}$, where $A_{0}$ is the cross-sectional area of the incident beam, $\theta_{0}$ is the angle the beam makes with the surface, and $\delta^{(2)}$ is the two-dimensional $\delta$ function. The quantity $A_{0} / \theta_{0}$ is the area of the surface intercepted by the incident beam. When the electron-density profile along the surface normal is a step function, the integration over $z$ yields $1 / Q_{z}^{2}=1 /(2 \theta k)^{2}$. The differential cross section becomes

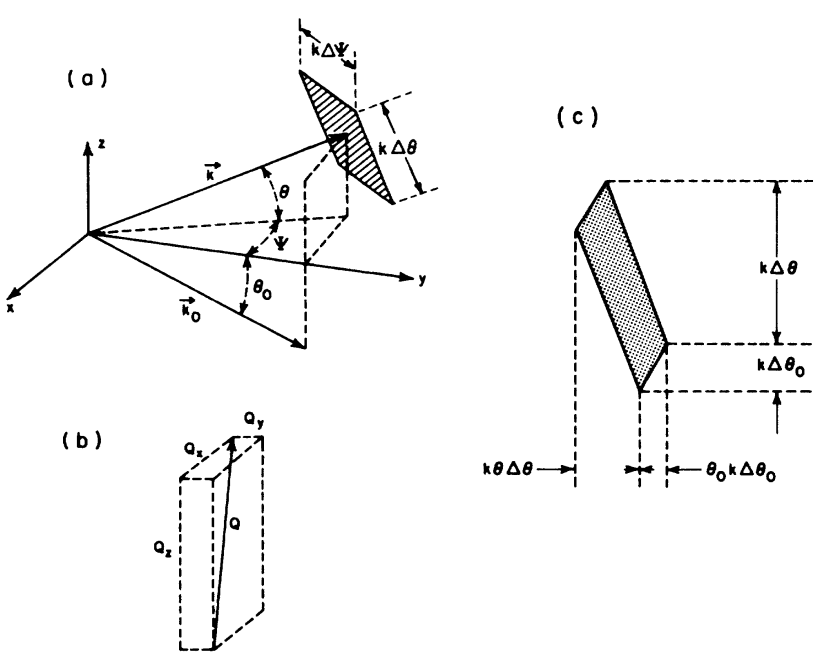

FIG. 3. Kinematics and resolution volume for a two-slit spectrometer. (a) Scattering kinematics, (b) wave-vector transfer, and (c) resolution volume.

$$
\frac{d \sigma}{d \Omega}=A_{0}\left(\frac{\theta_{c}^{2} k^{2}}{4 \pi}\right)^{2} \delta^{(2)}\left(\mathbf{Q}_{x y}\right)\left(\frac{\pi^{2}}{\theta^{3} k^{2}}\right) .
$$

With $d \Omega=d \theta d \psi=d^{2} \mathrm{Q}_{x y} /\left(k^{2} \theta\right)$, the integral over $d \Omega$ yields the total cross section

$$
\sigma=A_{0}\left(\frac{\theta_{c}}{2 \theta}\right)^{4} \text {. }
$$

Note that this calculation of the reflected intensity agrees with Eq. (2) in the limit of $\theta \gg \theta_{c}$, with $R_{F}(\theta)=\sigma / A_{0} \approx\left(\theta_{c} / 2 \theta\right)^{4}$.

Consider a flat surface that has a gradual density profile instead of a sharp boundary. The factor $1 / Q_{z}^{2}=1 /(2 \theta k)^{2}$ resulting from the integration over $d z$ is now replaced with (integrating by parts)

$$
\begin{aligned}
\left|\frac{1}{\rho_{e}(\infty)} \int d z e^{-i Q_{z} z} \rho_{e}(z)\right|^{2} & \\
& =\frac{1}{Q_{z}^{2}}\left|\frac{1}{\rho_{e}(\infty)} \int d z \frac{\partial \rho_{e}(z)}{\partial z} e^{-i Q_{z} z}\right|^{2} \\
& \equiv \frac{1}{Q_{z}^{2}}\left|\Phi\left(Q_{z}\right)\right|^{2}
\end{aligned}
$$

where $\rho_{e}(\infty)$ is the average electron density in the bulk of the material far from the surface. Thus $\left|\Phi\left(Q_{z}\right)\right|^{2}$ provides a measure of the sharpness of the interface profile. The predicted reflectivity is thus given by

$$
\frac{R\left(Q_{z}\right)}{R_{F}\left(Q_{z}\right)}=\left|\Phi\left(Q_{z}\right)\right|^{2}
$$

This expression has been derived by Beckmann and Spizzichino $^{15}$ for the general problem of reflection of electromagnetic radiation from rough surfaces and has been invoked by others in relation to optical reflectivity from the diffuse interface between two fluids near a critical point. ${ }^{16}$

A more realistic approximation to the nonideal surface is to assume that it has the same local, gradual surface profile everywhere, but that its height varies in the $x-y$ plane as some function $h\left(\mathbf{r}_{x y}\right)$. Interchanging the order of the $x-y$ and $z$ integrations in Eq. (7) results in

$$
\begin{aligned}
\frac{d \sigma}{d \Omega}= & \left(\frac{\theta_{c}^{2} k^{2}}{4 \pi Q_{z}}\right)^{2}\left|\Phi\left(Q_{z}\right)\right|^{2} \\
& \times\left|\int d^{2} \mathbf{r}_{x y} e^{-i Q_{x y} \cdot \mathbf{r}_{x y}} e^{-i Q_{z} h\left(\mathbf{r}_{x y}\right)}\right|^{2} .
\end{aligned}
$$

The differential cross section can be reexpressed as

$$
\frac{d \sigma}{d \Omega}=\left(\frac{\theta_{c}^{2} k^{2}}{4 \pi Q_{z}}\right)^{2}\left|\Phi\left(Q_{z}\right)\right|^{2}\left(\frac{A_{0}}{\theta}\right) X\left(\mathbf{Q}_{x y}, Q_{z}\right),
$$

where

$$
\begin{aligned}
X\left(\mathbf{Q}_{x y}, Q_{z}\right) & \equiv\left(\frac{\theta}{A_{0}}\right)\left|\int d^{2} \mathbf{r}_{x y} e^{-i \mathbf{Q}_{x y} \cdot \mathbf{r}_{x y}} e^{i Q_{z} h\left(\mathbf{r}_{x y}\right)}\right|^{2} \\
& =\int d^{2} \mathbf{r}_{x y} e^{-i Q_{x y} \cdot \mathbf{r}_{x y}}\left\langle e^{i Q_{z}\left[h\left(\mathbf{r}_{x y}\right)-h(0)\right]}\right\rangle .
\end{aligned}
$$




\section{Gaussian approximation}

The integral in Eq. (12) can be evaluated in terms of the height-height correlation function ${ }^{17}$ in the limit that the Hamiltonian is harmonic. This assumption is clearly valid for simple liquids where the coefficient of the quadratic term contains only the effects of gravity and of surface tension. In this approximation,

$$
\begin{aligned}
\left\langle e^{-i Q_{z}\left[h\left(\mathbf{r}_{x y}\right)-h(0)\right]}\right\rangle & =e^{-(1 / 2) Q_{z}^{2}\left\langle\left[h\left(\mathbf{r}_{x y}\right)-h(0)\right]^{2}\right\rangle} \\
& \equiv e^{-Q_{z}^{2} \sigma^{2}\left(r_{x y}\right)},
\end{aligned}
$$

where $r_{x y} \equiv\left|\mathbf{r}_{x y}\right|$ and $\sigma^{2}\left(r_{x y}\right) \equiv \frac{1}{2}\left\langle\left[h\left(\mathbf{r}_{x y}\right)-h(0)\right]^{2}\right\rangle$. If the height-height correlation function $\left\langle h\left(\mathbf{r}_{x y}\right) h(0)\right\rangle$ only has short-range correlations, $X\left(\mathbf{Q}_{x y}, Q_{z}\right)$ can be expressed as the sum of two terms

$$
X\left(\mathbf{Q}_{x y}, Q_{z}\right)=\left\langle X\left(\mathbf{Q}_{x y}, Q_{z}\right)\right\rangle+\delta X\left(\mathbf{Q}_{x y}, Q_{z}\right),
$$

where

$$
\begin{aligned}
\left\langle X\left(\mathbf{Q}_{x y}, Q_{z}\right)\right\rangle & =4 \pi^{2} \delta^{(2)}\left(\mathbf{Q}_{x y}\right)\left\langle e^{i Q_{z}\left[h\left(\mathrm{r}_{x y}\right)-h(0)\right]}\right\rangle \\
& =4 \pi^{2} \delta^{(2)}\left(\mathbf{Q}_{x y}\right) e^{-Q_{z}^{2} \sigma^{2}(\infty)},
\end{aligned}
$$

and $\sigma^{2}(\infty)=\left\langle h(0)^{2}\right\rangle$. The two-dimensional $\delta$ function $\delta^{(2)}\left(\mathbf{Q}_{x y}\right)$ makes the incident and reflected angles equal and in the same plane-the conditions for specular reflection. The second term $\delta X\left(Q_{x y}, Q_{z}\right)$ gives rise to diffuse scattering associated with surface roughness. The relationship between this term and the diffuse scattering intensity is presented in the Appendix.

The final result for the specular term is obtained by integrating $R(\theta)=A_{0}^{-1} d \sigma / d \Omega$ over the solid angle accepted by the detector $\Delta \Omega$. Taking $d^{2} \mathrm{Q}_{x y} / d \Omega=k^{2} \theta$,

$$
\begin{aligned}
R(\theta) & =\left[\frac{\theta_{c}^{2} k^{2}}{4 \pi Q_{z}}\right]^{2}\left|\Phi\left(Q_{z}\right)\right|^{2} \frac{1}{\theta} \int_{\Delta \Omega} \frac{d^{2} \mathbf{Q}_{x y}}{k^{2} \theta}\left\langle X\left(\mathbf{Q}_{x y}, Q_{z}\right)\right\rangle \\
& =\left(\frac{\theta_{c}}{2 \theta}\right)^{4} \frac{1}{4 \pi^{2}}\left|\Phi\left(Q_{z}\right)\right|^{2} \int_{\Delta \Omega} d^{2} \mathbf{Q}_{x y}\left\langle X\left(\mathbf{Q}_{x y}, Q_{z}\right)\right\rangle .
\end{aligned}
$$

For $\theta \gg \theta_{c}$, using the expression in Eq. (17) for $\left\langle X\left(\mathbf{Q}_{x y}, Q_{z}\right)\right\rangle$, the preceding equation becomes ${ }^{18}$

$$
\frac{R\left(Q_{z}\right)}{R_{F}\left(Q_{z}\right)} \approx e^{-\sigma^{2}(\infty) Q_{z}^{2}}\left|\Phi\left(Q_{z}\right)\right|^{2} \text {. }
$$

A more complicated situation arises when $h\left(\mathbf{r}_{x y}\right)$ and $h(0)$ are correlated over large distances. The extreme example is a "rough surface" for which $\left\langle\left[h\left(\mathbf{r}_{x y}\right)-h(0)\right]^{2}\right\rangle$ diverges as $r_{x y} \rightarrow \infty .{ }^{19,20}$ Under these circumstances one cannot make an objective distinction between specular reflection and diffuse scattering at angles close to the specular condition. The apparent specular reflectivity then varies with the resolution of the spectrometer.

The most general way to describe the measured reflectivity at a particular $\mathbf{Q}=\left(0,0, Q_{z}\right)$ is to define a resolution function $\Xi_{Q_{z}}\left(\mathbf{Q}-\mathbf{Q}^{\prime}\right)$ such that the measured reflectivity is the convolution

$$
R(\mathbf{Q})=\int d^{3} \mathbf{Q}^{\prime} S\left(\mathbf{Q}^{\prime}\right) \Xi_{Q_{z}}\left(\mathbf{Q}-\mathbf{Q}^{\prime}\right)
$$

where $\mathbf{Q}$ is the nominal position of the spectrometer and $S\left(\mathbf{Q}^{\prime}\right)$ is the scattering function. If $g\left(\theta_{0}\right) d \theta_{0}$ represents the angular distribution of the incident beam, normalized as $\int g\left(\theta_{0}\right) d \theta_{0}=1$, and $G(\theta, \psi) d \theta d \psi$ is the acceptance distribution of the detector, normalized as $G\left(\theta_{0}, 0\right)=1$, the measured reflectivity can be written as

$R(\mathbf{Q})=\iiint d \theta d \theta_{0} d \psi g\left(\theta_{0}\right) G(\theta, \psi) \frac{1}{A_{0}} \frac{d \sigma}{d \Omega}$.

If we write $Q_{x}=Q_{y}=0, Q_{z}=2 k \theta_{0}$ and $Q_{x}^{\prime}=k \psi$, $Q_{y}^{\prime}=k \theta_{0}\left(\theta_{0}-\theta\right)$, and $Q_{z}^{\prime}=k\left(2 \theta_{0}+\theta-\theta_{0}\right)$, and define $\phi \equiv \theta-\theta_{0}$, then close to the specular condition where $\phi \approx 0$,

$$
d^{3} Q^{\prime}=2 \theta_{0} k^{3} d \psi d \theta_{0} d \phi=2 \theta_{0} k^{3} d \psi d \theta_{0} d \theta .
$$

We can thus define

$S\left(\mathbf{Q}^{\prime}\right) \Xi_{Q_{z}}\left(\mathbf{Q}-\mathbf{Q}^{\prime}\right) \equiv \frac{d \theta_{0} d \Omega}{d^{3} \mathbf{Q}^{\prime}} g\left(\theta_{0}\right) G(\theta, \psi) \frac{1}{A_{0}} \frac{d \sigma}{d \Omega}$,

with

$$
S\left(\mathbf{Q}^{\prime}\right) \equiv\left(\frac{\theta_{c}}{2 \theta_{0}}\right)^{4} \frac{1}{4 \pi^{2}}\left|\Phi\left(Q_{z}^{\prime}\right)\right|^{2} X\left(\mathbf{Q}_{x y}^{\prime}, Q_{z}^{\prime}\right)
$$

and

$$
\Xi_{Q_{z}}\left(\mathbf{Q}-\mathbf{Q}^{\prime}\right) \equiv \frac{g\left(\theta_{0}\right) G(\theta, \phi)}{2 k} .
$$

The experimental geometry is illustrated schematically in Fig. 3. In Fig. 4, the $Q_{x}^{\prime}-Q_{z}^{\prime}$ cross section of the resolution function $\Xi_{Q_{z}}\left(\mathbf{Q}-\mathbf{Q}^{\prime}\right)$ is shown for an incident beam with intensity uniformly distributed throughout a range of angles $\Delta \theta_{0}$ and for a detector with uniform sensitivity over a range $\Delta \theta$, i.e., an open slit in front of a broad detector. $^{21}$ [The detector-limited resolution function in Fig. 4(b) is typical of the geometries used in the present experimental studies. See Table II.] If the spectrometer is tuned to the specular condition, then $\psi=0$ and $\theta=\theta_{0}$ at the center of the resolution volume.

Since nonvanishing values of $\Xi_{Q_{z}}\left(\mathbf{Q}-\mathbf{Q}^{\prime}\right)$ are restricted to $Q_{z}-Q_{z}^{\prime}<<Q_{z}$, and since both $\Phi\left(Q_{z}\right)$ and $X\left(\mathbf{Q}_{x y}, Q_{z}\right)$ are slowly varying functions of $Q_{z}$,

$$
\begin{aligned}
S\left(\mathbf{Q}^{\prime}\right) \Xi_{Q_{z}}\left(\mathbf{Q}-\mathbf{Q}^{\prime}\right)= & \frac{1}{4 \pi^{2}} R_{F}\left(Q_{z}\right)\left|\Phi\left(Q_{z}\right)\right|^{2} \\
& \times X\left(\mathbf{Q}_{x y}^{\prime}, Q_{z}\right) \Xi_{Q_{z}}\left(\mathbf{Q}-\mathbf{Q}^{\prime}\right) .
\end{aligned}
$$

Substitution of Eqs. (14) and (26) into Eq. (20) yields

$$
\begin{aligned}
& R(\mathbf{Q}) \approx \frac{1}{4 \pi^{2}} R_{F}\left(Q_{z}\right)\left|\Phi\left(Q_{z}\right)\right|^{2} \\
& \times \int d Q_{z}^{\prime} \int d^{2} \mathbf{Q}_{x y}^{\prime} \int d^{2} \mathbf{r}_{x y} e^{-i \mathbf{Q}_{x y}^{\prime} \cdot \mathbf{r}_{x y}} \\
& \times\left\langle e^{i Q_{z}\left[h\left(\mathrm{r}_{x y}\right)-h(0)\right]}\right\rangle \\
& \times \Xi_{Q_{z}}\left(\mathbf{Q}-\mathbf{Q}^{\prime}\right) .
\end{aligned}
$$


(a)

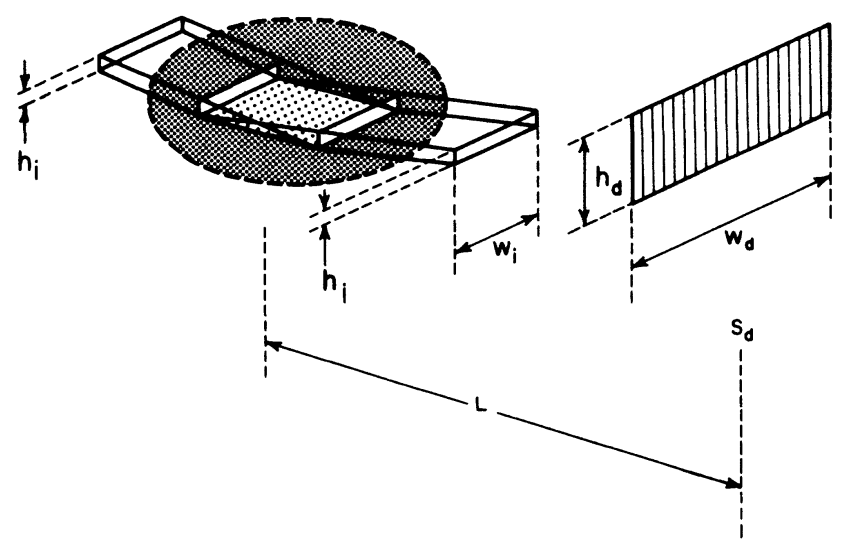

(b)

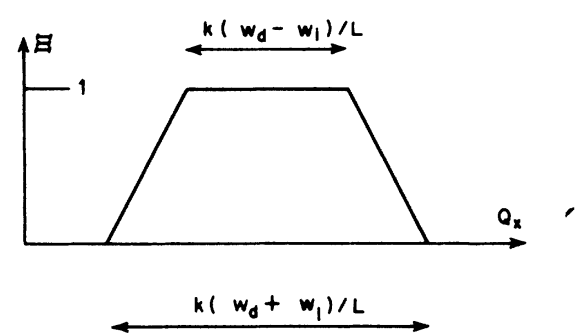

FIG. 4. Spectrometer geometry and resolution cross section. (a) Two-slit experimental geometry. (b) Trapezoidal resolution function.

We define

$\widetilde{\Xi}_{Q_{z}}\left(\mathbf{r}_{x y}, z=0\right) \equiv \int d Q_{z}^{\prime} \int d^{2} \mathbf{Q}_{x y}^{\prime} e^{-i \mathbf{Q}_{x y}^{\prime} \cdot \mathbf{r}_{x y}} \Xi_{Q_{z}}\left(\mathbf{Q}-\mathbf{Q}^{\prime}\right)$,

so that

$$
\begin{gathered}
R(\mathbf{Q}) \approx \frac{1}{4 \pi^{2}} R_{F}\left(Q_{z}\right)\left|\Phi\left(Q_{z}\right)\right|^{2} \\
\times \int d^{2} \mathbf{r}_{x y}\left\langle e^{i Q_{z}\left[h\left(\mathbf{r}_{x y}\right)-h(0)\right]}\right\rangle \\
\times \widetilde{\Xi}_{Q_{z}}\left(\mathbf{r}_{x y}, z=0\right) .
\end{gathered}
$$

Using Eqs. (19) and (20), the roughness $\sigma_{C}$ is defined by the relation

$e^{-Q_{z}^{2} \sigma_{C}^{2}}=\frac{1}{4 \pi^{2}} \int d^{2} \mathbf{r}_{x y}\left\langle e^{i Q_{z}\left[h\left(\mathbf{r}_{x y}\right)-h(0)\right]}\right\rangle \widetilde{\Xi}_{Q_{z}}\left(\mathbf{r}_{x y}, z=0\right)$.

Using the identity $\langle\exp (i \phi)\rangle=\exp \left(-\frac{1}{2}\left\langle\phi^{2}\right\rangle\right)$ and the previous definition for $\sigma^{2}\left(r_{x y}\right)$, the roughness is just the weighted average given by

$e^{-Q_{z}^{2} \sigma_{C}^{2}}=\frac{1}{4 \pi^{2}} \int d^{2} \mathbf{r}_{x y} e^{-Q_{z}^{2} \sigma^{2}\left(r_{x y}\right)} \widetilde{\Xi}_{Q_{z}}\left(\mathbf{r}_{x y}, z=0\right)$.

Alternatively, if the order of integration and statistical averaging can be interchanged,

$e^{-Q_{z}^{2} \sigma_{C}^{2}}=\frac{1}{4 \pi^{2}} \int d^{2} \mathbf{r}_{x y}\left\langle e^{i Q_{z}\left[h\left(\mathbf{r}_{x y}\right)-h(0)\right]} \widetilde{\Xi}_{Q_{z}}\left(\mathbf{r}_{x y}, z=0\right)\right\rangle$,

and if $\widetilde{\Xi}_{Q_{z}}\left(\mathbf{r}_{x y}, z=0\right)$ can be approximated by a Gaussian distribution, we obtain

$\sigma_{C}^{2}=\frac{1}{8 \pi^{2}} \int d^{2} \mathbf{r}_{x y}\left\langle\left[h\left(\mathbf{r}_{x y}\right)-h(0)\right]^{2}\right\rangle \widetilde{\Xi}_{Q_{z}}\left(\mathbf{r}_{x y}, z=0\right)$.

Defining the Fourier transform,

$$
h\left(\mathbf{r}_{x y}\right) \equiv \frac{1}{2 \pi} \int d^{2} \mathbf{Q}_{x y}^{\prime} \widetilde{h}\left(\mathbf{Q}_{x y}^{\prime}\right) e^{i \mathbf{Q}_{x y}^{\prime} \cdot \mathbf{r}_{x y}},
$$

it follows that

$$
\begin{aligned}
& \frac{1}{2}\left\langle\left[h\left(\mathbf{r}_{x y}\right)-h(0)\right]^{2}\right\rangle \\
& =\frac{1}{4 \pi^{2}} \int d^{2} \mathbf{Q}_{x y}^{\prime}\left\langle\left|\widetilde{h}\left(\mathbf{Q}_{x y}^{\prime}\right)\right|^{2}\right\rangle\left[1-\cos \left(\mathbf{Q}_{x y}^{\prime} \cdot \mathbf{r}_{x y}\right)\right] \\
& \quad \times \widetilde{\Xi}_{Q_{z}}\left(\mathbf{r}_{x y}, z=0\right) .
\end{aligned}
$$

If the order of integration is interchanged and the integral over $\mathbf{r}_{x y}$ is done first, and we make use of the definition

$$
\begin{gathered}
\Xi_{Q_{z}}\left(\mathbf{Q}_{x y}^{\prime}, z=0\right) \equiv \frac{1}{4 \pi^{2}} \int d^{2} \mathbf{r}_{x y} \widetilde{\Xi}_{Q_{z}}\left(\mathbf{r}_{x y}, z=0\right) \\
\times e^{i \mathbf{Q}_{x y}^{\prime} \cdot \mathbf{r}_{x y}},
\end{gathered}
$$

TABLE II. Parameters characterizing the various spectrometers used in this work. $\Delta Q_{z}=(4 \pi \lambda)\left(h_{d} / L\right)$ is the full width at half maximum of the resolution function $\Xi_{Q_{z}}\left(\mathbf{Q}-\mathbf{Q}^{\prime}\right)$ in the plane of incidence, where $L$ is the distance from sample to detector slit. The height-by-width dimensions of the incident slit and the detector slit define the resolution of the spectrometer. The $\mathrm{x}$-ray wavelength is $\lambda$. Different spectrometer configurations were used for $\mathrm{CCl}_{4}$ measurements in order to demonstrate the resolution dependence of the capillary-wave model.

\begin{tabular}{ccccccc}
\hline \hline Sample & $\begin{array}{c}\text { Spectrometer } \\
\text { resolution }\end{array}$ & $\begin{array}{c}\text { Monochromator } \\
\text { crystal }\end{array}$ & $\begin{array}{c}\Delta Q_{z} \\
\left(\AA^{-1}\right)\end{array}$ & $\begin{array}{c}h_{i} \times w_{1} \\
(\mathrm{~mm})\end{array}$ & $\begin{array}{c}h_{d} \times w_{d} \\
(\mathrm{~mm})\end{array}$ & $\begin{array}{c}\lambda \\
(\AA)\end{array}$ \\
\hline $\mathrm{H}_{2} \mathrm{O}$ & low & $\mathrm{Ge}(111)$ & 0.0124 & $0.5 \times 0.6$ & $1 \times 2$ & 1.529 \\
$\mathrm{CCl}_{4}$ & high & $\mathrm{Si}(111)$ & 0.0025 & $0.04 \times 0.7$ & $0.2 \times 1$ & 1.529 \\
$\mathrm{CCl}_{4}$ & high & $\mathrm{Ge}(111)$ & 0.0025 & $0.04 \times 0.7$ & $0.2 \times 1$ & 1.529 \\
$\mathrm{CCl}_{4}$ & low & $\mathrm{Ge}(111)$ & 0.0124 & $0.2 \times 0.7$ & $1 \times 1$ & 1.529 \\
$\mathrm{CH}_{3} \mathrm{OH}$ & low & $\mathrm{Ge}(111)$ & 0.0247 & $0.3 \times 6$ & $2 \times 18$ & 1.695 \\
\hline
\end{tabular}


then

$$
\begin{aligned}
\sigma_{C}^{2}=\int & d^{2} \mathbf{Q}_{x y}^{\prime}\left\langle\left|\widetilde{h}\left(\mathbf{Q}_{x y}^{\prime}\right)\right|^{2}\right\rangle \\
& \times\left[\Xi_{Q_{z}}\left(\mathbf{Q}_{x y}^{\prime}=0, z=0\right)-\Xi_{Q_{z}}\left(\mathbf{Q}_{x y}^{\prime}, z=0\right)\right]
\end{aligned}
$$

However, since

$$
\begin{aligned}
\Xi_{Q_{z}}\left(\mathbf{Q}_{x y}^{\prime}=0, z=0\right) & =\int d Q_{z}^{\prime} \Xi_{Q_{z}}\left(\mathbf{Q}-\mathbf{Q}^{\prime}\right) \\
& \left.\equiv \int 2 k d \theta_{0} \frac{g\left(\theta_{0}\right) G(\theta, \psi)}{2 k}\right|_{\theta=\theta_{0}, \psi=0}
\end{aligned}
$$

is normalized to unity, the final result for the roughness is

$$
\sigma_{C}^{2}=\int d^{2} \mathbf{Q}_{x y}^{\prime}\langle| \widetilde{h}\left(\left.\mathbf{Q}_{x y}^{\prime}\right|^{2}\right\rangle\left[1-\Xi_{Q_{z}}\left(\mathbf{Q}_{x y}^{\prime}, z=0\right)\right] \text {. }
$$

Since $1-\Xi_{Q_{z}}\left(Q_{x y}^{\prime}, z=0\right)=0$ for $\left|Q_{x y}^{\prime}\right|$ contained within the resolution volume as illustrated in Fig. 5, this result says that long-wavelength Fourier components of the surface roughness do not affect the measured reflectivity. One way to understand this is to note that when the surface is not flat, the decrease of reflectivity in the specular direction can be viewed as the sum of the destructive interference between waves reflected from different points. With a spectrometer of finite resolution, interference effects cannot be observed between points that are too far apart. Thus the measured $\sigma_{C}^{2}$ is the square average of the height differences between points that are separated by less than the appropriate coherence length of the radiation. With increasingly coarser resolution, the region in $\left|\mathbf{Q}_{x y}^{\prime}\right|$ over which $1-\Xi_{Q_{z}}\left(\mathbf{Q}_{x y}^{\prime}, z=0\right) \approx 0$ becomes larger and larger, and the integral yields smaller and smaller values of $\sigma_{C}^{2}$.

\section{CAPILLARY-WAVE MODEL}

The two-dimensional energy density for surface waves is made up of a surface tension term $(\gamma / 2)\left[\nabla_{x y} h\left(\mathbf{r}_{x y}\right)\right]^{2}$ and a gravitational term (important only at long wavelengths)

$$
U=\frac{\gamma}{2} \int d^{2} \mathbf{r}_{x y}\left|\nabla_{x y} h\left(\mathbf{r}_{x y}\right)\right|^{2}+\frac{\rho g}{2} \int d^{2} \mathbf{r}_{x y} h\left(\mathbf{r}_{x y}\right)^{2} .
$$

With the definition

$$
h\left(\mathbf{r}_{x y}\right) \equiv \frac{1}{2 \pi} \int d^{2} \mathbf{Q}_{x y} \tilde{h}\left(\mathbf{Q}_{x y}\right) e^{i \mathbf{Q}_{x y} \cdot \mathbf{r}_{x y}},
$$

the total energy can also be written as

$$
U=\frac{1}{2} \int d^{2} \mathbf{Q}_{x y}\left(\gamma\left|\mathbf{Q}_{x y}\right|^{2}+\rho g\right) \widetilde{h}\left(\mathbf{Q}_{x y}\right)^{2} .
$$

Taking into account the density of states and applying the equipartition theorem,

$$
\frac{k_{B} T}{2}=\frac{1}{2}\left(\gamma\left|\mathbf{Q}_{x y}\right|^{2}+\rho g\right) 4 \pi^{2}\left\langle\widetilde{h}\left(\mathbf{Q}_{x y}\right)^{2}\right\rangle,
$$

one obtains the following expression for the mean-

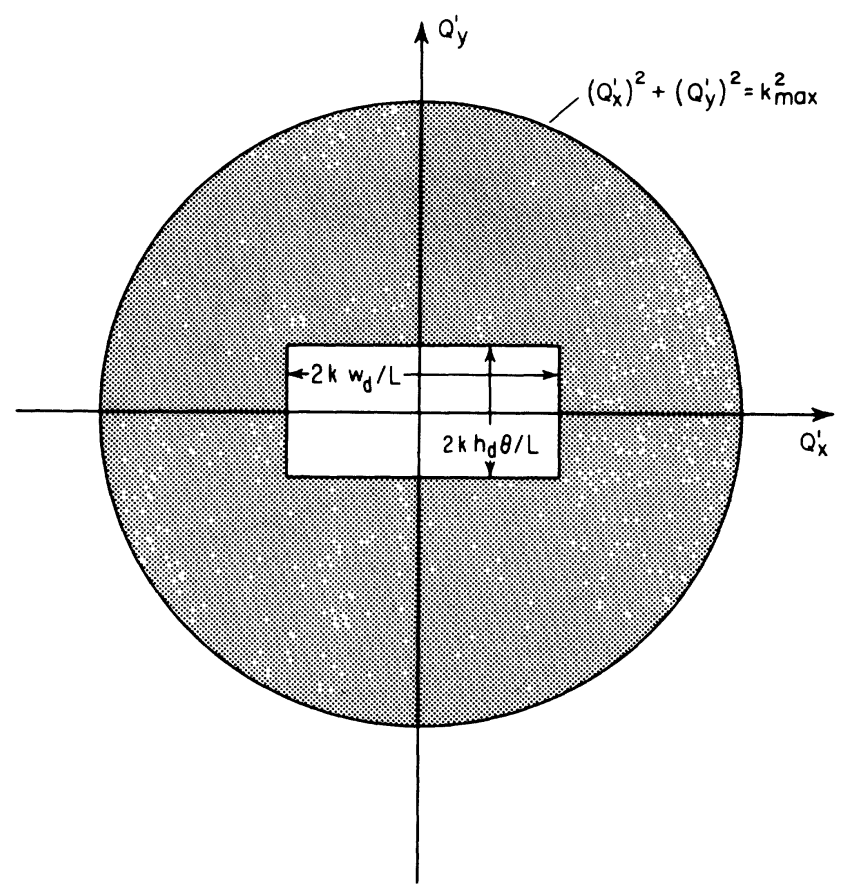

FIG. 5. Interception of the resolution volume with the $\mathbf{Q}_{x y}^{\prime}$ plane for a small incident beam slit and a large detector slit. The measured roughness arises from capillary-wave modes that scatter the reflected beam outside of the resolution volume of the detector. The integration in Eq. (46) is over the shaded area.

squared roughness of the free surface due to capillary waves,

$$
\left\langle h(0)^{2}\right\rangle=\frac{k_{B} T}{4 \pi^{2} \gamma} \int d^{2} \mathbf{Q}_{x y} \frac{1}{\left|\mathbf{Q}_{x y}\right|^{2}+k_{g}^{2}},
$$

where $k_{g}^{2} \equiv \rho g / \gamma$.

The upper limit of this integral $k_{\max }$ is on the order of $\pi / r_{M}$, where $r_{M}$ is the molecular radius. Without the gravity term $\rho g / \gamma$, the integral would have an "infrared" divergence since as $\mathbf{Q}_{x y} \rightarrow 0$ the energy associated with Fourier components $\widetilde{h}\left(\mathbf{Q}_{x y}\right)$ vanishes. Using these limits, integration of Eq. (44) results in the following equation for the "intrinsic" roughness of a liquid surface,

$$
\left\langle h(0)^{2}\right\rangle=\frac{k_{B} T}{4 \pi \gamma} \ln \left(\frac{k_{\max }^{2}+k_{g}^{2}}{k_{g}^{2}}\right) .
$$

For water $\left\langle h(0)^{2}\right\rangle^{1 / 2} \approx 3.98 \AA$ at room temperature. ${ }^{22}$ We will presently show that the gravitational length scale $\left(1 / k_{g}\right)$ is inaccessible with realistic resolution volumes, and the measured or effective roughness is larger than the intrinsic roughness.

Substitution of Eq. (43) into Eq. (39) yields

$$
\begin{aligned}
\sigma_{C}^{2}=\frac{k_{B} T}{4 \pi^{2} \gamma} \int & d^{2} \mathbf{Q}_{x y}\left(\frac{1}{\left|\mathbf{Q}_{x y}\right|^{2}+k_{g}^{2}}\right) \\
& \times\left[1-\Xi_{Q_{z}}\left(\mathbf{Q}_{x y}, z=0\right)\right],
\end{aligned}
$$

where $\sigma_{C}$ is the effective root-mean-squared roughness 
due to capillary waves. Since $1-\Xi_{Q_{z}}\left(Q_{x y}, z=0\right) \approx 0$ for any $\mathbf{Q}_{x y}$ small enough for the gravity term to make a contribution, the $k_{g}$ term can be neglected.

The two-slit resolution function $\Xi_{Q_{z}}\left(\mathbf{Q}_{z y}, z=0\right)$ appropriate to the experiments discussed below is illustrated in Fig. 4. A uniformly illuminated rectangular slit just before the sample fixes the dimensions of the incident beam. In this geometry, the detector follows a second slit located a distance $L$ from the sample. The two slits have dimensions $h_{i} \times w_{i}$ and $h_{d} \times w_{d}$, respectively. The resultant resolution function has trapezoidal cross sections. Figure 4(b) shows $\Xi_{Q_{z}}\left(\mathbf{Q}-\mathbf{Q}^{\prime}\right)$ along the $Q_{x}$ direction. The flat top has a length along $Q_{z}$ of $2 k_{x, 1}=k\left(w_{d}-w_{i}\right) / L$ and a base of length $2 k_{x, 2}=k\left(w_{d}+w_{i}\right) / L$. From Fig. 3(c), one can see that the trapezoidal cross section in the $Q_{y}$ direction would have a top of length $2 k_{y, 1}=k \theta\left(h_{d}-h_{i}\right) / L$ and a base of length $2 k_{y, 2}=k \theta\left(h_{d}+h_{i}\right) / L$. Although the algebra is straightforward for this trapezoidal resolution function, for purposes of illustration it is easier to consider the case where the incident beam is much smaller than the detector slit. Then the trapezoid reduces to a rectangle as illustrated in Fig. 5. Since the measured $\left\langle h(0)^{2}\right\rangle$ arises from all of the modes which scatter the beam outside of the resolution volume, the integration is over the shaded area in Fig. 5. One of the two integrations in Eq. (46) can be done analytically, yielding the contribution to the measured roughness due to capillary waves,

$$
\begin{aligned}
\left\langle h(0)^{2}\right\rangle=\frac{k_{B} T}{2 \pi^{2} \gamma}[ & \pi \int_{0}^{k_{\max }} d Q_{r}\left(\frac{1}{Q_{r}^{2}+k_{g}^{2}}\right) \\
& -\int_{-k_{y}}^{+k_{y}} d Q_{y} \frac{1}{\left(Q_{y}^{2}+k_{g}^{2}\right)^{1 / 2}} \\
& \left.\times \tan ^{-1}\left(\frac{k_{x}}{\left(Q_{y}^{2}+k_{g}^{2}\right)^{1 / 2}}\right)\right] .
\end{aligned}
$$

The first term is the integral over the entire circle in Fig. 5 , i.e., the intrinsic roughness. The second term subtracts contributions from capillary modes that fall within the resolution volume. The second integral can be simplified by observing that $k_{x} \gg k_{y} \geq Q_{y}$ for all $Q_{y}$ (and $k_{x} \gg k_{g}$ ), with the result

$$
\tan ^{-1}\left[k_{x} /\left(Q_{y}^{2}+k_{g}^{2}\right)^{1 / 2}\right] \approx \pi / 2 .
$$

Thus the roughness is approximately given by

$$
\begin{aligned}
\left\langle h(0)^{2}\right\rangle \approx & \frac{k_{B} T}{2 \pi \gamma}\left[\log \left[\frac{\left(k_{\max }^{2}+k_{g}^{2}\right)^{1 / 2}}{k_{g}}\right]\right. \\
& \left.-\log \left[\frac{k_{y}+\left(k_{y}^{2}+k_{g}^{2}\right)^{1 / 2}}{k_{g}}\right]\right] \\
= & \frac{k_{B} T}{2 \pi \gamma}\left[\frac{\left(k_{\max }^{2}+k_{g}^{2}\right)^{1 / 2}}{k_{y}+\left(k_{y}^{2}+k_{y}^{2}\right)^{1 / 2}}\right] .
\end{aligned}
$$

Finally, since $k_{\max } \gg k_{y} \gg k_{g}$, the preceding expression reduces to

$$
\left\langle h(0)^{2}\right\rangle \approx \frac{k_{B} T}{2 \pi \gamma} \log \left(\frac{k_{\max }}{2 k_{y}}\right) .
$$

Note that neither the gravity term $k_{g}$ nor the width of the detector $k_{x}$ appears in this final result.

In this approximation, the reflectivity $R\left(Q_{z}\right)$, obtained by replacing the value of $\sigma^{2}$ in Eq. (19) by $\sigma_{C}^{2}$ is

$$
R\left(Q_{z}\right)=\left|\Phi\left(Q_{z}\right)\right|^{2} e^{-\sigma_{C}^{2}\left(Q_{z}\right) Q_{z}^{2}}
$$

where

$$
\begin{aligned}
\sigma_{C}^{2}\left(Q_{z}\right) Q_{z}^{2} & =\frac{8 \pi k_{B} T \theta^{2}}{\lambda^{2} \gamma} \ln \left[\frac{\lambda}{2 \theta\left(\Delta \theta_{d}\right) r_{M}}\right) \\
& =\frac{k_{B} T}{2 \pi \gamma} Q_{z}^{2} \ln \left(\frac{2 \pi}{Q_{z} r_{M}\left(\Delta \theta_{d}\right)}\right),
\end{aligned}
$$

and $r_{M}$ is the molecular radius and $\Delta \theta_{d}=h_{d} / L$ is the full width of the angular resolution in the plane of incidence. It is particularly important to note the logarithmic dependence on $Q_{z}$. As mentioned above, the quantity $\left|\Phi\left(Q_{z}\right)\right|$ describes the local diffuse character of the liquid-vapor interface. Although various forms have been proposed for the profile of this interface, ${ }^{16}$ the data do not justify anything beyond the simplest Gaussian form, $\left|\Phi\left(Q_{z}\right)\right|=\exp \left(-\frac{1}{2} Q_{z}^{2} \sigma_{P}^{2}\right)$. The final form for the reflectivity is thus

$$
\frac{R\left(Q_{z}\right)}{R_{F}\left(Q_{z}\right)}=e^{-\sigma_{T}^{2}\left(Q_{z}\right) Q_{z}^{2}},
$$

where

$$
\sigma_{T}^{2}\left(Q_{z}\right) Q_{z}^{2} \approx\left[\sigma_{P}^{2}+\sigma_{C}^{2}\left(Q_{z}\right)\right] Q_{z}^{2}
$$

combines the effect of the profile $\left(\sigma_{P}\right)$ with the effects of capillary waves $\left[\sigma_{C}\left(Q_{z}\right)\right]$.

\section{EXPERIMENTAL METHOD}

The data were obtained at two locations. Measurements of water and carbon tetrachloride were performed at synchrotron beam line D4 at HASYLAB (Ref. 23) in Hamburg, West Germany, while the methanol experiments were done at beam line X22B at the National Synchrotron Light Source (NSLS) at Brookhaven National Laboratory.

The major technical challenge in building an $x$-ray spectrometer for the study of the free surface of liquids is the limitation that the sample must be kept horizontal. At synchrotrons, this requires a means of bending the horizontal beam downward. The spectrometer used at HASYLAB functions by tilting the monochromator crystal to direct the $x$-ray beam down at varying incident angles to the liquid surface. ${ }^{3,24}$ Because the monochromator crystal at beam line D4 is illuminated by the "white" synchrotron beam, the bending is accomplished by a simple tilt of the monochromator goniometer. The size of the incident beam is determined by a slit located before the monochromator crystal. The slight shift in the $x$-ray wavelength selected by the monochromator due to the 
small change in angle from this tilt is not important because the reflected intensity is normalized to the incident intensity using a beam monitor located after the monochromator. The liquid sample is mounted on an elevator that is lowered as the monochromator is tilted. Since the sample is centered between the monochromator and the detector, the x-ray beam is specularly reflected into a detector that is at the same height as the monochromator. A slit before the detector determines the outgoing resolution.

The spectrometer used at NSLS differs from previous synchrotron liquid-surface spectrometers in the method of tilting the horizontal x-ray beam. After Bragg reflection from a monochromator crystal, the beam is bent down onto the liquid sample by a second matched perfect crystal that is rotated about the beam incident on this crystal. The rotation of the second crystal causes the beam to sweep out a cone with a vertical deflection of $\alpha=(2 \pi / \lambda) \sin \theta_{B} \sin \chi$ and a horizontal shift of $\Delta \theta=(2 \pi / \lambda) \sin \theta_{B}(1-\cos \chi)$, where $\theta_{B}$ is the Bragg angle of the crystal reflection and $\chi$ is the rotation angle. $\mathrm{A}$ rotation about the axis of the incoming beam has the advantage of eliminating the small shift in the $x$-ray energy produced in the other spectrometers when the monochromator is tilted about an axis perpendicular to its reciprocal lattice vector. This rotation is necessary in a spectrometer using two "monochromator" crystals, but can also be employed in a design using a single crystal. At bean line $\mathrm{X} 22 \mathrm{~B}$, the second, matched crystal is mounted in the center of a standard, horizontally scattering, Huber 4-circle diffractometer. The entire liquid freesurface spectrometer is mounted on the " $2 \theta$ " arm of the Huber diffractometer. The liquid sample cell is located on an elevator centered $600 \mathrm{~mm}$ from the $2 \theta$ axis. Symmetric input and output arms hold vacuum flight paths and defining slits. Other than the preceding differences, for the purposes of the experiments reported here, the spectrometer was similar to the instrument used at HASYLAB. A detailed description of the spectrometer and its use will appear elsewhere. ${ }^{25}$

In all of these experiments, the liquid sample is spread on a Pyrex disk placed inside a sealed cell. The Pyrex disk is thoroughly cleaned at a temperature of $\sim 100^{\circ} \mathrm{C}$ either in chromic-sulfuric acid or, preferably, in an equal mixture of sulfuric acid with concentrated (30\%) hydrogen peroxide, ${ }^{26}$ so that the liquid completely wets the glass surface. The disks had a diameter of $57 \mathrm{~mm}$ in our first cells; a second design permitted the use of a larger area sample with 76-mm-diam. Pyrex disks. In the larger cell, about $1.4 \mathrm{ml}$ of liquid is used to form a uniform layer about $300 \mu \mathrm{m}$ high. The use of a sample this thin is critical in order to damp out the long-wavelength vibrations that would otherwise obscure the measurements. However, the samples were thick enough to avoid reflection from the glass substrate as absorption limits beam penetration to depths to less than $25 \mu \mathrm{m}$ at the angles of incidence used. The $\mathrm{x}$-ray windows of the sample cell were made on Kapton. A $1000 \AA$ gold layer was deposited on the windows to improve their thermal homogeneity with the rest of the oven. The gold coating reduces condensation on the windows - important when using vola- tile liquids such as carbon tetrachloride and methanol. The experiments reported here were all performed at room temperature.

\section{EXPERIMENTAL RESULTS}

\section{A. Water}

$X$-ray reflectivity measurements of the free surface of water (surface tension of $73 \mathrm{dyne} / \mathrm{cm}$ ) have been previously reported. ${ }^{3}$ These results are reproduced in Fig. 6. The measured reflectivity is plotted versus $Q_{z}$ as closed circles in Fig. 6(a). The solid line is the reflectivity given by the Fresnel law of optics. At small wave vectors the agreement between the Fresnel law and the data is excellent. However, at the largest wave vectors the difference becomes significant. It is the deviation from the Fresnel law which allows us to measure the actual roughness of the interface.

The capillary-wave model, Eq. (52), predicts that the ratio of the measured reflectivity divided by the Fresnel reflectivity $R / R_{F}$ should be nearly Gaussian. Figure 7 is a semilog plot of this ratio versus $Q_{z}^{2}$. The data points fall on a straight line. The solid line is the theoretical prediction given by Eq. (53) with $\sigma_{P}=0$, that is, including only the contribution from capillary waves. The slight upward curvature results from the logarithmic term in Eq. (51). The effective roughness from capillary waves $\sigma_{C}$ depends weakly on $Q_{z}$ as predicted by Eq. (51)

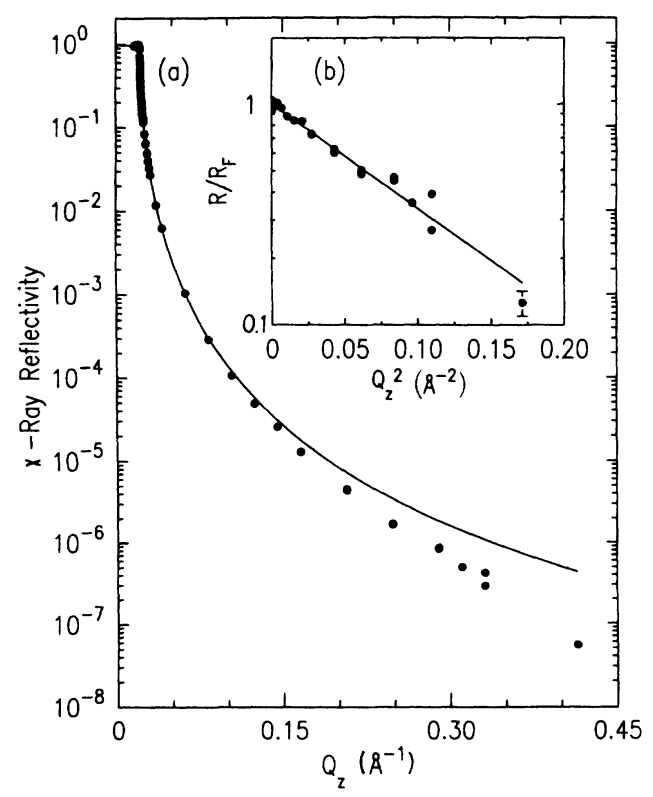

FIG. 6. Measured x-ray reflectivity of the free surface of water. (a) X-ray reflectivity as a function of the longitudinal wave-vector transfer. The solid line is the calculated Fresnel reflectivity for the ideal step-function interface. (b) Logarithm of the measured reflectivity normalized to the Fresnel reflectivity plotted vs the square of the wave-vector transfer. The solid line is the best fit to the form $R\left(Q_{z}\right) / R_{F}=\exp \left(-\sigma_{M}^{2} Q_{z}^{2}\right)$, where $\sigma_{M}=3.3 \pm 0.1 \AA$. 


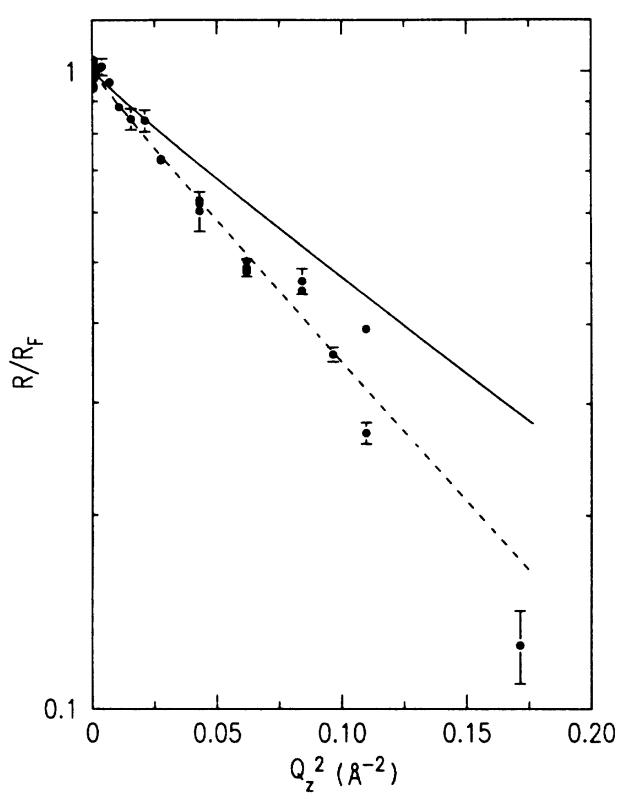

FIG. 7. Experimental results and the capillary-wave model for water. Measured reflectivity from the water-free surface normalized to the Fresnel reflectivity is plotted vs the square of the wave-vector transfer. The solid line is the prediction of the capillary-wave model for the spectrometer configuration used in the experiment. The dashed line is the best fit to Eq. (53) giving a surface profile width of $\sigma_{P}=1.8 \pm 0.2 \AA$, approximately equal to the expected radius of the water molecule (1.93 $\AA$ ).

and shown in Table III. A fit to Eq. (53) where only the profile width is allowed to vary gives $\sigma_{P}=1.8 \pm 0.2 \AA$ (Ref. 27) as shown by the dashed line in Fig. 7. The fitted profile width is reasonably close to the average radius of the water molecule of $1.93 \AA$. We have also fit the data to a simple Gaussian form, $\exp \left(-\sigma_{M}^{2} Q_{z}^{2}\right)$, where the result $\sigma_{M}=3.30 \AA$ includes the effects of both the profile and capillary waves. ${ }^{28}$

\section{B. Carbon tetrachloride}

The surface tension of carbon tetrachloride $\left(\mathrm{CCl}_{4}\right)$, $\gamma=27$ dyne $/ \mathrm{cm}$, is nearly three times smaller than that of water. Therefore the root-mean-squared amplitude for thermal capillary waves should be larger by approximately a factor of 1.6. In Fig. 8, the ratio $R / R_{F}$ is shown for $\mathrm{CCl}_{4}$ (see Table II for the spectrometer configurations) and for water. The reflectivity for $\mathrm{CCl}_{4}$ is considerably smaller than that of water, as shown in the figure. At the largest $q$ vectors the ratio of the reflectivity for these two liquids is consistent with the ratio of the two surface tensions. This is somewhat fortuitous since the ratio is only applicable to $\sigma_{C}$ and not $\sigma_{T}$.

In order to determine the resolution dependence of the capillary-wave model, different spectrometer configurations were used for the $\mathrm{CCl}_{4}$ measurements. Reflectivity data for the "high" and "low" resolution configurations (see Table II) as shown in Fig. 9 as closed circles and triangles. The capillary-wave model predicts that as the resolution is coarsened, for example, by enlarging the detector slit, there should be an increase in the number of capillary-wave modes which are accepted by the detector. The reflectivity data with the coarser resolution are higher, as predicted by the capillary-wave model. Since the resolution enters logarithmically into $\sigma_{C}$ the resolution must be changed dramatically to observe this effect. For the measurements of interest this amounts to about a $20 \%$ decrease in the roughness for a factor of 5 increase in the resolution (see Tables II and III).

The results of the capillary-wave model with $\sigma_{P}=0$ for the two different spectrometer configurations are shown in Fig. 9 as solid lines. The capillary-wave contribution $\sigma_{C}$ varies from 4.5 to $5.0 \AA$ for $\mathrm{CCl}_{4}$ and depends on the resolution and the $q$ vector as shown in Table III. As is the case for water, for $\mathrm{CCl}_{4}$ the capillary-wave model is not sufficient to explain the reflectivity data. It is necessary to add a profile width $\sigma_{P}$ to model the observed reflectivity. We have fit the deviation from the Fresnel law to the form $\exp \left[-\sigma_{T}^{2}\left(Q_{z}\right) Q_{z}^{2}\right]$, where $\sigma_{T}^{2}=\sigma_{C}^{2}+\sigma_{P}^{2}$ and only $\sigma_{P}$ is allowed to vary. The fitted profile width depends on the spectrometer and varies from $3.9 \pm 0.1$ to $2.5 \pm 0.1$ for the different configurations. The values of the measured profile width $\sigma_{P}$ are close to the molecular radius of $3.88 \AA$ for $\mathrm{CCl}_{4}$. The spread in the measured values of $\sigma_{P}$ may be related to systematic uncertainties.

We have also fit the data to a simple exponential form $\exp \left(-\sigma_{M}^{2} Q_{z}^{2}\right)$. The fitted width $\sigma_{M}$ varies from 5.13 to $6.04 \AA$. The results are summarized in Table III.

TABLE III. Theoretical and experimental results for the roughness of the studied liquids. $\sigma_{C}\left(Q_{z}\right)$ is the value calculated from Eq. (51) for various values of $Q_{z}$. The measured quantity $\sigma_{M}$ is obtained by the best fit of the data to the form $\exp \left(-\sigma_{M}^{2} Q_{z}^{2}\right)$, while $\sigma_{P}$ is from the best fit to the general form, Eq. (51), using the listed values of the molecular radius $r_{M}$, and the surface tension $\gamma$. The spectrometer configuration used for each of the measurements is given in Table II. Different spectrometer configurations were used for $\mathrm{CCl}_{4}$ measurements in order to demonstrate the resolution dependence of the capillary-wave model.

\begin{tabular}{|c|c|c|c|c|c|c|c|}
\hline Sample & $\begin{array}{l}r_{M} \\
(\AA)\end{array}$ & $\begin{array}{c}\gamma \\
\text { (dyne/cm) }\end{array}$ & $Q_{z}=0.2 \AA^{-1}$ & $\begin{array}{c}\sigma_{C}\left(Q_{z}\right) \\
(\AA) \\
Q_{z}=0.3 \AA^{-1} \\
\end{array}$ & $Q_{z}=0.4 \AA^{-1}$ & \multicolumn{2}{|c|}{ (Measured) } \\
\hline $\mathrm{H}_{2} \mathrm{O}$ & 1.93 & 73 & 2.88 & 2.82 & 2.77 & $3.3 \pm 0.1$ & $1.8 \pm 0.2$ \\
\hline $\mathrm{CCl}_{4}$ & & & 5.00 & 4.90 & 4.83 & $5.93 \pm 0.06$ & $3.9 \pm 0.1$ \\
\hline $\mathrm{CCl}_{4}$ & 3.38 & 27 & 5.00 & 4.90 & 4.83 & $6.04 \pm 0.07$ & $3.3 \pm 0.1$ \\
\hline $\mathrm{CCl}_{4}$ & & & 4.59 & 4.48 & 4.41 & $5.13 \pm 0.06$ & $2.5 \pm 0.1$ \\
\hline $\mathrm{CH}_{3} \mathrm{OH}$ & 2.52 & 23 & 4.85 & 4.73 & 4.65 & $4.80 \pm 0.06$ & $0.7 \pm 0.4$ \\
\hline
\end{tabular}




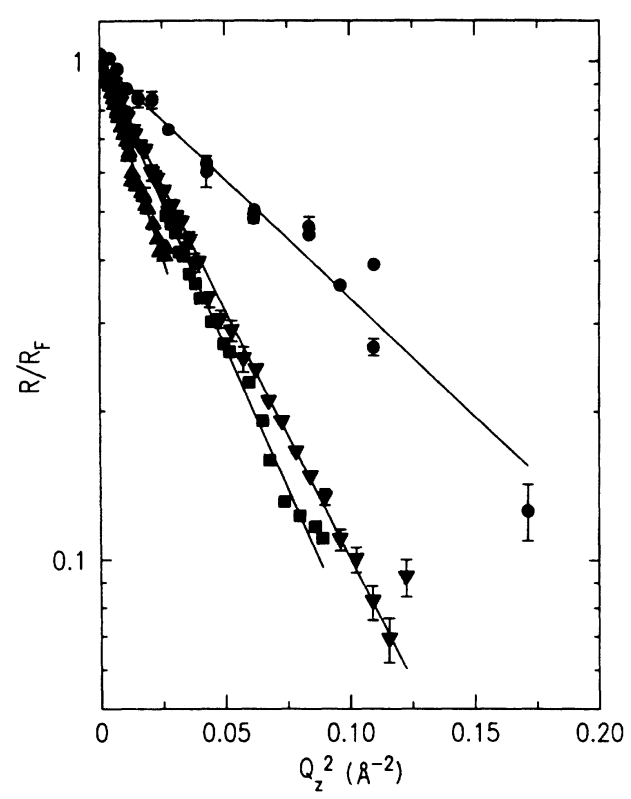

FIG. 8. Measured x-ray reflectivities. The measured reflectivities normalized to the Fresnel reflectivities are plotted vs $Q_{z}^{2}$. The closed triangles (closed squares) are data for carbon tetrachloride from a high (low)-resolution spectrometer. The closed circles are the water data repeated from a previous figure. The inverted closed triangles are data for the free surface of methanol. The solid line is the best fit to the form $R\left(Q_{z}\right) / R_{F}=\exp \left(-\sigma_{M}^{2} Q_{z}^{2}\right)$.

\section{Methanol}

The surface tension of methanol $\left(\mathrm{CH}_{3} \mathrm{OH}\right), \gamma=23$ dyne/cm, is $15 \%$ smaller than that of $\mathrm{CCl}_{4}$, and we expected that the reflectivity would also be slightly lower. In Fig. 10, the reflectivity $R / R_{F}$ is plotted versus $Q_{z}^{2}$. Although the data look rather similar to the $\mathrm{CCl}_{4}$ data a quantitative analysis reveals a fundamental difference. The solid line in Fig. 10 displays the theoretical reflectivity using the form $\exp \left(-\sigma_{C}^{2} Q_{z}^{2}\right)$ that only includes the contribution from capillary waves. Over the range from $Q_{z}=0.2$ to $0.4 \AA^{-1}, \sigma_{C}$ varies from 4.85 to $4.65 \AA$ as shown in Table III. In contrast to the other two liquids, the capillary-wave model with $\sigma_{P}=0$ is very close to the measured value. If we allow $\sigma_{P}$ to vary, the best fit is achieved with $\sigma_{P}=0.7 \pm 0.4$. This value is considerably smaller than the molecular radius $r_{M}=2.52 \AA$, suggesting the molecular radius may be an inappropriate measure of the surface-profile width. Since the electron density tensor is rather anisotropic for methanol, surface ordering of the methanol molecule could significantly reduce $\sigma_{P}$ from $r_{M}$. However, we do not believe that this effect alone can explain the methanol data.

Another possibility is that the capillary-wave model, which incorporates a sharp cutoff of the modes at $\pi / r_{M}$, may be incorrect at small length scales. ${ }^{29}$ The justification of the cutoff is that capillary waves cannot propagate on length scales smaller than the molecular size. However, our capillary model, which uses a step function cutoff, assumes the

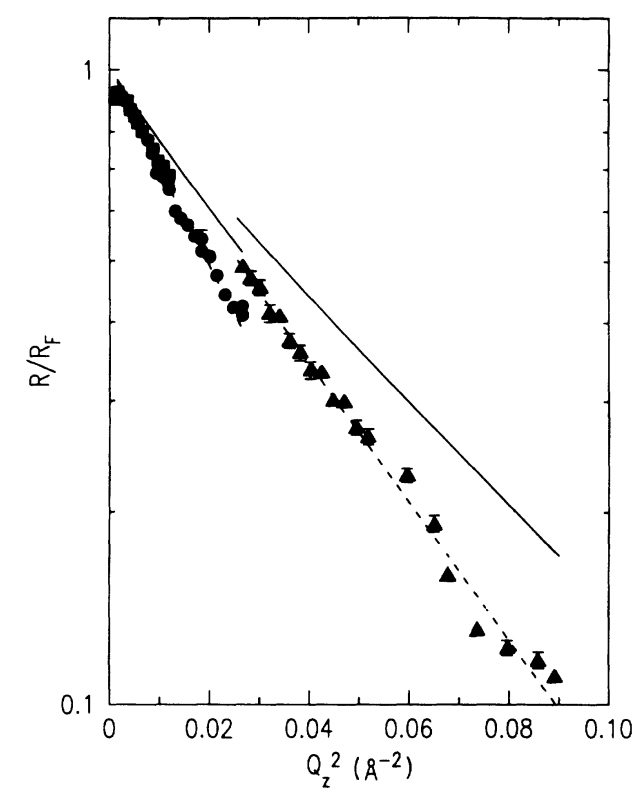

FIG. 9. Reflectivity of carbon tetrachloride. The measured reflectivity of carbon tetrachloride normalized to the Fresnel reflectivity is shown. The closed squares are data from a "high" resolution spectrometer and a $\mathrm{Si}(111)$ monochromator crystal. The closed circles are for a "high" resolution spectrometer and a Ge(111) monochromator crystal. The closed triangles are for a "low" resolution spectrometer and a Ge(111) monochromator crystal. The straight lines are the predictions of the capillarywave model for the two resolutions. The dashed lines are the best fit to a surface-profile width which is added in quadrature to the capillary roughness. The results of the fits are summarized in Table III.

$$
(\gamma / 2)\left[\nabla_{x y} h\left(\mathbf{r}_{x y}\right)\right]^{2}
$$

term is constant at all length scales. If, for instance, there is an enhancement in the surface tension at large values of $\mathbf{Q}_{x y}$, the capillary-wave contribution would be smaller. This enhancement seems plausible since the methanol molecule is anisotropic relative to both water and $\mathrm{CCl}_{4}$. If, for instance, the cutoff was raised from $\pi / r_{M}$ to $2 \pi / r_{M}, \sigma_{C}$ is reduced by approximately $0.2 \AA$, which in turn increases $\sigma_{P}$ to approximately $1.5 \AA$. Therefore the possibility exists that the deduced value of $\sigma_{P}$ is wrong because we have incorrectly estimated the proper way to sum over the thermal capillary modes.

\section{CONCLUSION}

For the measurements presented above for water, carbon tetrachloride, and methanol, the continuum model for thermally induced capillary waves on the free surface is a reasonable description of the roughness. The data clearly show that there is an additional contribution to the roughness due to the finite size of the molecules. In the case of water, and also for carbon tetrachloride, this length is identified as the radius of the molecule. However, for methanol, which is rather anisotropic relative to both water and to carbon tetrachloride, this length is 


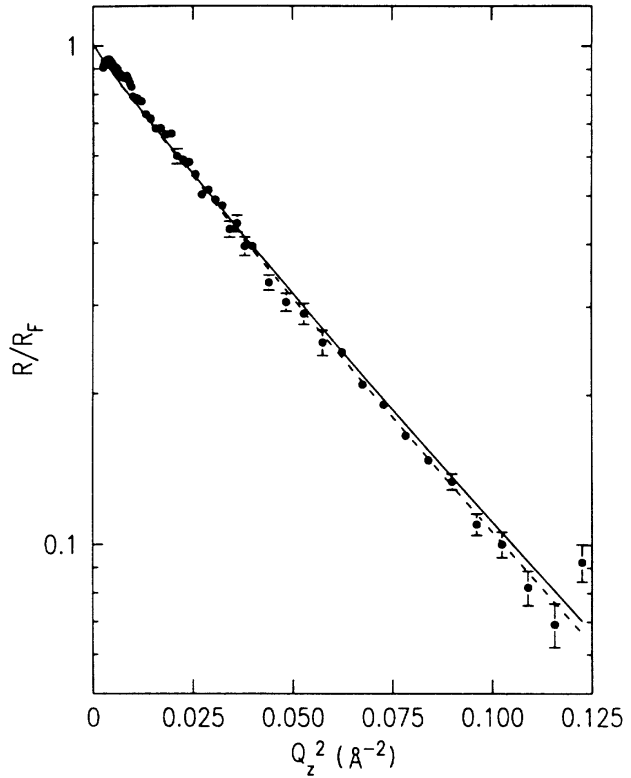

FIG. 10. Reflectivity of methanol. The measured x-ray reflectivity normalized to the Fresnel reflectivity from methanol is plotted as a function of $Q_{z}^{2}$. The solid line is the prediction of the capillary-wave model with no adjustable parameters. The dashed line is the result of a least-squares fit to a profile width which is added in quadrature to the capillary-wave roughness. The resulting width $\sigma=0.7 \pm 0.1 \AA$ suggests that the anisotropy of the methanol molecule must be considered. The spectrometer configuration is given in Table II.

smaller than the mean radius of the molecule suggesting that the profile width is smaller than the mean molecular radius. This could possibly be due to some degree of molecular orientation at the surface. Alternatively, it is also possible that the capillary-wave model is not valid at the smallest length scales and that the preceding calculation overestimates the thermal contribution to the roughness.

This work demonstrates that the technique of $x$-ray reflectivity is well suited for the study of the roughness of the liquid-vapor interface. However, the present work only includes measurements of diffuse scattering near the specular condition. Measurement of diffuse scattering away from the specular condition remains an interesting and challenging problem.

\section{ACKNOWLEDGMENTS}

We would like to thank all of the people who have helped in this series of experiments, performed at several laboratories around the world. In particular, we gratefully acknowledge the technical staff of the Ris $\varnothing$ National Laboratory who assisted at HASYLAB, D. Schwartz who assisted at Harvard and NSLS, and J. Axe and the staff of the Physics Department of the Brookhaven National Laboratory. This work was supported in part by the Harvard Materials Science Laboratory through U.S. National Science Foundation Grant Nos. DMR-86-14003 and DMR-85-13523, the Joint Services Electronics Program through Grant No. N00014-84-K-0465, the Danish National Science Foundation, and the Ris $\varnothing$ National Laboratory. Research carried out at the National Synchrotron Light Source, Brookhaven National Laboratory, is supported by the Department of Energy Division of Material Science and Division of Chemical Sciences under Contract No. DE-AC02-76CH00016.

\section{APPENDIX: DIFFUSE SCATTERING}

The diffuse $x$-ray scattering from a rough surface can be treated as a perturbation on the solutions for a smooth surface. We model the polarizability of our rough surface by a surface that is ideally flat and smooth, coated with an infinitesimally thin surface layer having an $x$-ray polarizability given by

$$
\alpha\left(\mathbf{r}_{x y}\right)=\left(\frac{r_{e} \lambda^{2} \rho_{e}}{4 \pi^{2}}\right) h\left(\mathbf{r}_{x y}\right)=\left(\frac{\theta_{c}^{2}}{4 \pi}\right) h\left(\mathbf{r}_{x y}\right),
$$

where $h\left(\mathbf{r}_{x y}\right)$ is the height of the surface at $\mathbf{r}_{x y}$. This expression is only valid in the limit that $q h \ll 1$. For a plane wave $\mathbf{E}^{i}\left(\theta_{0}\right)$ incident at an angle $\theta_{0}$ with respect to the surface plane, ${ }^{30}$ the surface electric field $\mathbf{E}^{s}\left(\theta_{0}\right)$ is the sum of the incident field $\mathbf{E}^{i}\left(\theta_{0}\right)$ and the reflected field $\mathbf{E}^{r}\left(\theta_{0}\right)$ that are determined by solutions to the boundary value problem for the ideal flat surface. The surface electric field $\mathbf{E}^{s}\left(\theta_{0}\right)$ acts on the inhomogeneous polarizability to induce a surface current distribution,

$$
\mathbf{J}\left(\mathbf{r}_{x y}\right)=i(\omega / c) \alpha\left(\mathbf{r}_{x y}\right) \mathbf{E}^{s}\left(\theta_{0}\right) e^{-i \mathbf{k}^{l} \cdot \mathbf{r}_{x y}},
$$

where $\mathbf{J}$ and $\mathbf{E}$ are polarized in the plane of the surface and $\mathbf{k}^{i}$ is the incident wave vector. If $\alpha\left(\mathbf{r}_{x y}\right)$ and $\mathbf{J}\left(\mathbf{q}_{x y}\right)$ are defined in terms of their Fourier integrals in the plane of the surface, with

$$
\alpha\left(\mathbf{r}_{x y}\right)=\frac{1}{2 \pi} \int d^{2} \mathbf{q}_{x y} \alpha\left(\mathbf{q}_{x y}\right) e^{i \mathbf{q}_{x y} \cdot \mathbf{r}_{x y}}
$$

and

$$
\mathbf{J}\left(\mathbf{q}_{x y}\right)=\frac{1}{2 \pi} \int d^{2} \mathbf{r}_{x y} \mathbf{J}\left(\mathbf{r}_{x y}\right) e^{-i \mathbf{q}_{x y} \cdot \mathbf{r}_{x y}},
$$

then

$$
\mathbf{J}\left(\mathbf{q}_{x y}\right)=i(\omega / c) \alpha\left(\mathbf{q}_{x y}+\mathbf{k}^{i}\right) \mathbf{E}^{s}\left(\theta_{0}\right) .
$$

The surface current $\mathbf{J}\left(\mathbf{q}_{x y}\right)$ induces transmitted and reflected fields that modulate the incoming and outgoing fields at the surface, respectively. We will refer to these induced fields as diffuse transmitted $(d t)$ and diffuse reflected $(d r)$. The diffuse transmitted wave goes as

$$
\mathbf{E}^{d t} \exp \left[i\left(\mathbf{q}_{x y} \cdot \mathbf{r}_{x y}+\mathbf{k}^{d t} \cdot \hat{\mathbf{z}}\right)\right],
$$

and the diffuse reflected wave goes as

$$
\mathbf{E}^{d r} \exp \left[i\left(\mathbf{q}_{x y} \cdot \mathbf{r}_{x y}-\mathbf{k}^{d r} \cdot \hat{\mathbf{z}}\right)\right],
$$

where $\mathbf{k}^{d t} \cdot \hat{\mathbf{z}}=-(\omega / c) \theta$ and $\mathbf{k}^{d r} \cdot \hat{\mathbf{z}}=(\omega / c)\left(\theta^{2}-\theta_{c}^{2}\right)^{1 / 2}$ are wave-vector components along the surface normal. Assuming a thin surface transition layer, the amplitudes of the surface fields can be obtained from the continuity 
equations for the tangential components of $\mathbf{E}$ and $\mathbf{H}$. For simplicity, we consider only the case where the electric field is polarized parallel to the surface and normal to the plane of incidence, since for small angles the general result is independent of the incident polarization. For a thin current layer normal to the plane of incidence $\mathbf{J}\left(\mathbf{q}_{x y}\right)$, the continuity of $\mathbf{E}$ and $\mathbf{H}=\nabla \times \mathbf{E}$ gives

$$
E_{x}^{d r}\left(\mathbf{q}_{x y}\right)=E_{x}^{d t}\left(\mathbf{q}_{x y}\right)
$$

and

$$
k_{z}^{d r} E_{x}^{d r}\left(\mathbf{q}_{x y}\right)+k_{z}^{d t} E_{x}^{d t}\left(\mathbf{q}_{x y}\right)=\frac{4 \pi}{c} J_{x}\left(\mathbf{q}_{x y}\right),
$$

where the solution to these equations yields

$$
E^{d r}\left(\mathbf{q}_{x y}\right)=\frac{(4 \pi i \omega / c) \alpha\left(\mathbf{q}_{x y}+\mathbf{k}^{i}\right) E^{s}\left(\theta_{0}\right)}{\theta+\left(\theta^{2}-\theta_{c}^{2}\right)^{1 / 2}} .
$$

The autocorrelation function for the scattered field is given by

$$
\begin{aligned}
\left\langle E^{d r}\left(\mathbf{r}_{x y}\right) E^{d r}(0)\right\rangle= & \frac{4 \pi \omega / c}{\theta+\left(\theta^{2}-\theta_{c}^{2}\right)^{1 / 2}} \\
& \times\left\langle\alpha\left(\mathbf{r}_{x y}\right) \alpha(0)\right\rangle e^{-i \mathbf{k}^{\prime} \cdot \mathbf{r}_{x y}} \\
& \times\left|\mathbf{E}^{s}\left(\theta_{0}\right)\right|^{2},
\end{aligned}
$$

or in terms of the height-height correlation function as

$$
\begin{aligned}
\left\langle E^{d r}\left(\mathbf{r}_{x y}\right) E^{d r}(0)\right\rangle= & \frac{\left(\theta_{c}^{2} \omega\right) / c}{\theta+\left(\theta^{2}-\theta_{c}^{2}\right)^{1 / 2}} \\
& \times\left\langle h\left(\mathbf{r}_{x y}\right) h(0)\right\rangle e^{-i \mathbf{k}^{\prime} \cdot \mathbf{r}_{x y}} \\
& \times\left|E^{s}\left(\theta_{0}\right)\right|^{2} .
\end{aligned}
$$

Since the electric field is enhanced at the surface by

$$
E^{s}\left(\theta_{0}\right)=\frac{2 \theta_{0}}{\theta_{0}+\left(\theta_{0}^{2}-\theta_{0}^{2}\right)^{1 / 2}} E^{i}\left(\theta_{0}\right) .
$$

the autocorrelation function can be expressed in terms of the incident field

$$
\begin{aligned}
\left\langle E^{d r}\left(\mathbf{r}_{x y}\right) E^{d r}(0)\right\rangle= & \left(2 \theta_{0} \omega / c\right)^{2}\left[R_{F}(\theta) R_{F}\left(\theta_{0}\right)\right]^{1 / 2} \\
& \times\left\langle h\left(\mathbf{r}_{x y}\right) h(0)\right\rangle e^{-i \mathbf{k}^{i} \cdot \mathbf{r}_{x y}} \\
& \times\left|E^{i}\left(\theta_{0}\right)\right|^{2},
\end{aligned}
$$

where $R_{F}$ is the Fresnel expression given by Eq. (3).

It is straightforward to calculate the far-field pattern corresponding to these surface fields from the Kirchoff integral

$$
\mathbf{A}(\mathbf{r})=\int_{\mathbf{r}^{\prime}=\mathbf{r}_{x y}^{\prime}} d^{2} \mathbf{r}_{x y}^{\prime} \cdot \nabla^{\prime} G\left(\mathbf{r} ; \mathbf{r}^{\prime}\right) \mathbf{A}\left(\mathbf{r}^{\prime}\right)
$$

using a Green's function that vanishes on the surface, ${ }^{31}$

$$
G\left(\mathbf{r} ; \mathbf{r}^{\prime}\right)=\frac{1}{4 \pi}\left[\frac{e^{-i \omega R / c}}{R}-\frac{e^{-i \omega R^{\prime} / c}}{R^{\prime}}\right),
$$

where $R^{\prime}$ is the mirror image of $R=\left|\mathbf{r}-\mathbf{r}^{\prime}\right|$ in the plane of the surface. Noting that $k_{z}^{d r}=(\omega / c) \theta$, in the direction near to the specular reflection

$$
d^{2} \mathbf{r}^{\prime} \cdot \nabla G\left(\mathbf{r} ; \mathbf{r}^{\prime}\right) \approx d^{2} \mathbf{r}^{\prime}\left(\frac{\omega \theta}{2 \pi c}\right)\left[\frac{e^{-i \omega r / c+i \mathbf{k}^{d r} \cdot \mathbf{r}_{x y}}}{r}\right) .
$$

Since the illuminated sample area is given by $A_{0} / \theta_{0}$, the integral can be evaluated in the far field to obtain

$$
\begin{aligned}
|\mathbf{A}(\mathbf{r})|=\frac{A_{0}}{\theta_{0}}\left(\frac{\omega \theta}{2 \pi c r}\right) \int & d^{2} \mathbf{r}_{x y} e^{i \mathbf{k}^{\mathrm{d} r} \cdot \mathbf{r}_{x y}} \\
& \times\left\langle\mathbf{A}^{d r}\left(\mathbf{r}_{x y}\right) \mathbf{A}^{d r}(0)\right\rangle .
\end{aligned}
$$

It is straightforward to obtain

$\frac{d \sigma}{d \Omega}=\frac{A_{0}}{\theta_{0}}\left(\frac{\omega \theta}{2 \pi c}\right) \int d^{2} \mathbf{r}_{x y} e^{i \mathbf{k}^{\mathrm{d} r} \cdot \mathbf{r}_{x y}} \frac{\left\langle E^{d r}\left(\mathbf{r}_{x y}\right) E^{d r}(0)\right\rangle}{\left|E^{i}\left(\theta_{0}\right)\right|^{2}}$.

On substitution of the electric field correlation function, Eq. (65),

$$
\begin{aligned}
\frac{d \sigma}{d \Omega}= & \frac{A_{0}}{\theta_{0}} \frac{(\omega / c)^{4}}{4 \pi^{2}}\left\{\left(4 \theta \theta_{0}\right)^{2}\left[R_{F}(\theta) R_{F}\left(\theta_{0}\right)\right]^{1 / 2}\right\} \\
& \times \int d^{2} \mathbf{r}_{x y} e^{i\left(\mathbf{k}^{d r}-\mathbf{k}^{i}\right) \cdot \mathbf{r}_{x y}}\left\langle h\left(\mathbf{r}_{x y}\right) h(0)\right\rangle
\end{aligned}
$$

the differential cross section is given in terms of the height-height correlation function. We note that this form is also applicable to scattering from solids. The term

$$
\left(4 \theta \theta_{0}\right)^{2}\left[R_{F}(\theta) R_{F}\left(\theta_{0}\right)\right]^{1 / 2}
$$

is the "surface scattering enhancement factor." It is interesting to note that $\theta$ and $\theta_{0}$ appear symmetrically. For angles $\theta \gg \theta_{c}$ and $\theta_{0} \gg \theta_{c}$ the surface enhancement factor is unity. This is clearly not the case when $\theta$ or $\theta_{0}$ approach the critical angle. For instance, if both $\theta$ and $\theta_{0}$ are exactly equal to the critical angle then the scattering is enhanced by exactly 16. Although other general derivations of this result have been published and applied to radar and optical regions of the electromagnetic spectrum, ${ }^{32-35}$ we believe the relative simplicity of the present derivation for the small angles appropriate to the $\mathrm{X}$-ray problem is advantageous.

Although the existence of the surface scattering enhancement factor should prove useful in distinguishing surface scattering from bulk scattering, for simplicity we will only consider the region where the enhancement factor is unity. For capillary waves, the cross section for diffuse scattering becomes

$$
\frac{d \sigma}{d \Omega}=\left(\frac{A_{0}}{\theta_{0}}\right) \frac{(\omega / c)^{4}}{4 \pi^{2}}\left(\frac{k_{B} T}{\gamma\left|\mathbf{Q}_{x y}\right|^{2}+\rho g}\right),
$$

where $\mathbf{Q}_{x y}=\left(\mathbf{k}^{d r}-\mathbf{k}^{i}\right) \cdot \mathbf{r}_{x y}$. In the limit that $\theta$ and $\theta_{0} \gg \theta_{c}$,

$$
\frac{d \sigma}{d \Omega}=\left(r_{e} \rho\right)^{2}\left(\frac{A_{0}}{\theta_{0}}\right)\left(\frac{k_{B} T}{\gamma\left|\mathbf{Q}_{x y}\right|^{2}+\rho g}\right) .
$$




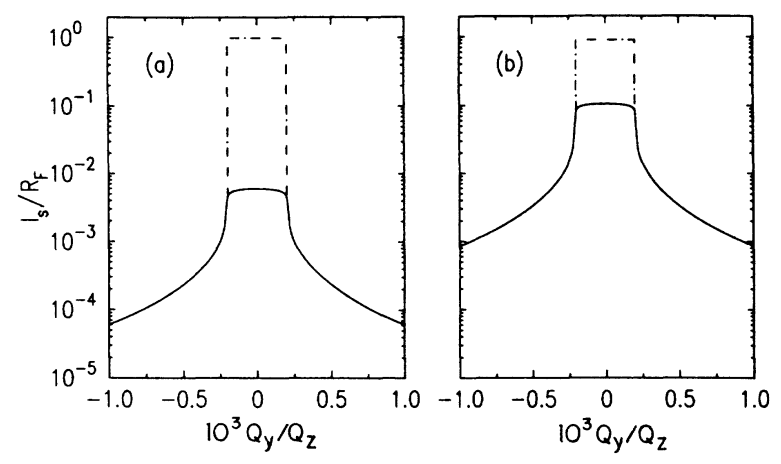

FIG. 11. Calculated diffuse $x$-ray scattering of the free surface of water. The scattering is calculated from Eq. (77) using the spectrometer parameters given in Table II. The transverse wave vector, $Q_{y}$, is normalized to $Q_{z}$ and the intensity is normalized to $R_{F}$. The dot-dashed line is the total intensity that would be observed as the spectrometer is scanned through the specular condition. (a) $Q_{z}=0.03 \AA^{-1}$. (b) $Q_{z}=0.10 \AA^{-1}$.

It is interesting to note that the final form of the differential cross section does not depend on $Q_{z}$.

The diffuse scattering intensity

$$
I_{D}=\frac{1}{A_{0}} \int_{\text {resolution }} \frac{d \sigma}{d \Omega} d \Omega
$$

involves an integration over the resolution volume. By converting to reciprocal space and normalizing $I_{D}$ to $R_{F}$ the integration reduces to a simple integral over the $q_{x}$ and $q_{y}$ resolution which is centered at $Q_{x}$ and $Q_{y}$,

$$
\frac{I_{D}\left(Q_{x}, Q_{y}, Q_{z}\right)}{R_{F}}=\sigma_{D}^{2} Q_{z}^{2},
$$

where

$$
\sigma_{D}^{2}=\frac{k_{B} T}{4 \pi^{2}} \int_{Q_{s}-k_{x}, Q_{y}-k_{y}}^{Q_{x}+k_{x}, Q_{y}+k_{y}} \frac{d q_{x} d q_{y}}{\gamma\left|\mathbf{Q}_{x y}+\mathbf{q}_{x y}\right|^{2}+\rho g},
$$

and the integral is over a region in $q_{x}-q_{y}$ like that shown in Fig. 5. Note that the width of the rectangle along $Q_{y}$ is linearly proportional to $Q_{z}$, that is, $2 k_{y} \approx 2 \theta k\left(h_{D} / L\right) \approx Q_{z}\left(h_{D} / L\right)$. This result is only valid in the limit that $Q_{z} \sigma_{D} \ll<1$ and does not contain a Debye-Waller Gaussian term since only single scattering events are considered in the analysis. At larger $q$ vectors, the correct result must contain a Debye-Waller term so that the scattering sum rule is satisfied. This is analogous to one-phonon process in three dimensions ${ }^{36}$

$$
\frac{I_{D}\left(Q_{x}, Q_{y}, Q_{z}\right)}{R_{F}}=\sigma_{D}^{2} Q_{z}^{2} e^{-\sigma_{T}^{2} Q_{z}^{2}},
$$

where $\sigma_{T}$ contains the effects of the profile and capillary waves as described in the text. ${ }^{37}$ The results of the calculated diffuse scattering for water, using the resolution given in Table II, are shown for $Q_{x}=0$ and for two values of $Q_{z}$ in Fig. 11. As discussed in the text, the total signal observed at the specular condition $\left(Q_{y} \approx 0\right)$ is the sum of the intrinsic specular reflectivity (due to the longwavelength gravitational cutoff) and the diffuse scattering that falls within the resolution function. The dashed line in Fig. 11 illustrates the predicted level for the total intensity observed as the spectrometer is scanned through the specular condition. Although the solid line in Fig. 11 depends on the gravitational cutoff, the total intensity, as indicated by the dashed line, is independent of the cutoff.

For short wave vectors $\left(Q_{z} \sigma_{T}<1\right)$, the diffuse scattering predicted by Eq. (75) increases with $Q_{z}$, both from the explicit dependence on $Q_{z}^{2}$ and from the implicit dependence associated with the increase of the projected height of the resolution function $k_{y}$ with $Q_{z}$. On the other hand, for the resolution shown in Fig. 5 and the parameters in Table II, one can demonstrate (and the numerical results in Fig. 5 illustrate), that when $I_{D}\left(0, Q_{y}, Q_{z}\right)$ is written as a function of $Q_{y} / Q_{z}$, the functional form is independent of $Q_{z}$.

In the limit $k_{y} \ll k_{x}$, the integral is approximately equal to

$$
\sigma_{D}^{2}=\left(\frac{k_{B} T}{4 \pi \gamma}\right)\left[\frac{\Delta Q_{y}+k_{y}}{\left|Q_{y}-k_{y}\right|}\right) .
$$

If $k_{y} \ll\left|Q_{y}\right|$, then

$$
\sigma_{D}^{2}=\left(k_{B} T / 4 \pi \gamma\right)\left(2 \Delta Q_{y} /\left|Q_{y}\right|\right) .
$$

If the spectrometer is tuned off the specular condition, then for water at room temperature and $k_{y} \ll\left|Q_{y}\right|$, the expression for the ratio of the scattering to the Fresnel reflectivity is given by

$\frac{I_{D}\left(0, Q_{y}, Q_{z}\right)}{R_{F}} \approx\left(0.66 Q_{z}\right)^{2}\left[\frac{2 k_{y}}{\left|Q_{y}\right|}\right] e^{\left(-3.3 Q_{z}\right)^{2}}$,

where $Q_{z}$ is in $\AA^{-1}$. For constant $2 k_{y} / Q_{y}, I_{D} / R_{F}$ is maximized at $0.2 \AA^{-1}$. At $0.2 \AA^{-1}$ and $2 k_{y} / Q_{y}=1$, $I_{D} / R_{F} \approx 0.01$, which corresponds to about 1 count $/ \mathrm{sec}$ at an incident flux of $10^{7}$ counts/sec and is clearly measurable at existing synchrotron sources.

A second practical issue associated with measuring weak diffuse scattering at angles close to the specular condition is whether or not the tails of the resolution function obscure the weak diffuse signal. For the conditions just described, the predicted ratio of the diffuse intensity to the specular signal is approximately $1.5 \%$. Spectrometers can be designed to detect this level.

A more serious technical challenge will be separating the surface scattering from the total scattering consisting of both surface and bulk scattering. For scattering angles close to the critical angle, the angular dependence associated with the surface enhancement factor may provide the means for separating the two signals. Another possibility is to use the wavelength dependence of the scattering, as the $\mathrm{x}$-ray penetration into the bulk varies as the third power of the wavelength. ${ }^{7}$ 
${ }^{1}$ J. Als-Nielsen, F. Christensen, and P. S. Pershan, Phys. Rev. Lett. 48, 1107 (1982).

${ }^{2}$ P. S. Pershan and J. Als-Nielsen, Phys. Rev. Lett. 52, 759 (1984).

${ }^{3}$ A. Braslau, M. Deutsch, P. S. Pershan, A. H. Weiss, J. AlsNielsen, and J. Bohr, Phys. Rev. Lett. 54, 114 (1985).

${ }^{4}$ S. A. Rice, Nature 316, 108 (1985).

${ }^{5}$ S. G. Wolf, L. Leiserowitz, M. Lahav, M. Deutsch, K. Kjaer, and J. Als-Nielsen, Nature 328, 63 (1987).

${ }^{6}$ K. Kjaer, J. Als-Nielsen, C. A. Helm, L. A. Laxhuber, and H. Mohwald, Phys. Rev. Lett. 58, 2224 (1987).

${ }^{7}$ P. S. Pershan, A. Braslau, A. H. Weiss, and J. Als-Nielsen, Phys. Rev. A 35, 4800 (1987).

${ }^{8}$ F. P. Buff, R. A. Lovett, and F. H. Stillinger, Jr., Phys. Rev. Lett. 15, 621 (1965).

9J. S. Huang and W. W. Webb, J. Chem. Phys. 50, 3677 (1969).

${ }^{10}$ S. R. Andrews and R. A. Cowley, J. Phys. C 18, 6427 (1985).

${ }^{11}$ I. K. Robinson, Phys. Rev. B 33, 3830 (1986).

12J. D. Jackson, Classical Electrodynamics (Wiley, New York, 1975), p. 281.

${ }^{13}$ See Ref. 12, p. 421.

${ }^{14}$ See Ref. 12, Eq. (9.106), p. 420.

${ }^{15} \mathrm{P}$. Beckmann and A. Spizzichino, The Scattering of Electromagnetic Waves from Rough Surfaces (MacMillan, New York, 1963).

${ }^{16}$ E. S. Wu and W. W. Webb, Phys. Rev. A 8, 2065 (1973).

${ }^{17}$ L. D. Landau and E. M. Lifshitz, Statistical Physics Part 1 (Pergamon, Oxford, 1980), p. 334.

${ }^{18}$ For angles close to $\theta_{c}\left(\theta>\theta_{c}\right)$, a WKB approximation yields $R\left(Q_{z}\right) / R_{F}\left(Q_{z}\right)=\exp \left[-\sigma^{2} Q_{z}\left(Q_{z}^{2}-Q_{c}^{2}\right)^{1 / 2}\right]$. Since this result is derived under the approximation $\sigma Q_{c} \ll 1$ the difference between it and Eq. (19) is of only minor importance. L. Sanders (private communication).

${ }^{19}$ C. A. Croxton, Statistical Mechanics of the Liquid Surface (Wiley, New York, 1980).

${ }^{20}$ F. S. Rys, Phys. Rev. Lett. 56, 624 (1986).

${ }^{21}$ All of the data presented below were taken with synchrotron beams having $\Delta \theta_{0} \approx m c^{2} / E=(1.5-2.0) \times 10^{-4}$, where $E$ is given in $\mathrm{GeV}$. In fact, the angular spread is further reduced by at least a factor of 10 by the slit just before the sample that defines the height of the beam.

${ }^{22} \mathrm{R}$. Loudon, in Surface Excitations, edited by V. M. Agranovich and R. Loudon (Elsevier, New York, 1984), p. 589.

${ }^{23}$ Hamburger Synchrotronstrahlungslabor, Deutsches Elecktronen-Synchrotron.

${ }^{24} \mathrm{~J}$. Als-Nielsen and P. S. Pershan, Nucl. Instrum. Methods 208, 545 (1983).

${ }^{25}$ A. Braslau, Ph.D. thesis, Harvard University, 1988.

${ }^{26}$ This "piranha" solution [S. Wasserman (private communication)] is preferable to the chromic-sulfuric acid solution which leads to a leaching of chromium ions.

${ }^{27}$ All of the errors include one standard deviation uncertainty from the fitting. In adddition, we have included an uncertainty of $5 \%$ in the absolute reflectivity scale at $0.30 \AA^{-1}$.

${ }^{28}$ The form, $\exp \left(-\sigma_{M}^{2} Q_{z}^{2}\right)$, which ignores the logarithmic correction was used in the previous publication. Since $\sigma_{C}$ only changes from 2.88 to $2.77 \AA$ in going from $Q_{z}=0.2$ to $0.4 \AA^{-1}$, this approximation is reasonable.

${ }^{29}$ J. Meunier, J. Phys. 48, 1819 (1987).

${ }^{30} \mathrm{We}$ use $\theta_{0}$ and $\theta$ to describe the incident and exit angles rather than $\alpha$ and $\beta$ since $\alpha$ is the standard measure of the polarizability.

${ }^{31}$ J. D. Jackson, Ref. 12, p. 429.

32J. M. Elson, Phys. Rev. B 12, 2541 (1975).

${ }^{33}$ D. B. Barrick, in Radar Cross Section Handbook (Plenum, New York, 1970).

${ }^{34}$ A. A. Maradudin, Phys. Rev. B 11, 1392 (1975).

${ }^{35}$ E. L. Church, H. A. Jenkinson, and J. M. Zavada, Opt. Eng. 16, 360 (1977).

${ }^{36}$ N. W. Ashcroft and N. D. Mı.rmin, Solid State Physics (Holt, Rinehart and Winston, New York, 1976), p. 794.

${ }^{37} \mathrm{~A}$ similar result can be obtained by considering the effect of increasing the resolution volume. The difference in the scattering for the different resolution volumes is the diffuse scattering from a resolution volume which is the difference of the original two resolution volumes. This technique for calculating the diffuse scattering differs from the result in the text whereby the term $\sigma_{D}^{2} Q_{z}^{2}$ is replaced by $1-\exp \left(\sigma_{D}^{2} Q_{z}^{2}\right)$. These results agree for small $Q_{z}$ but differ when $Q_{z} \sigma_{D}$ approaches unity. 


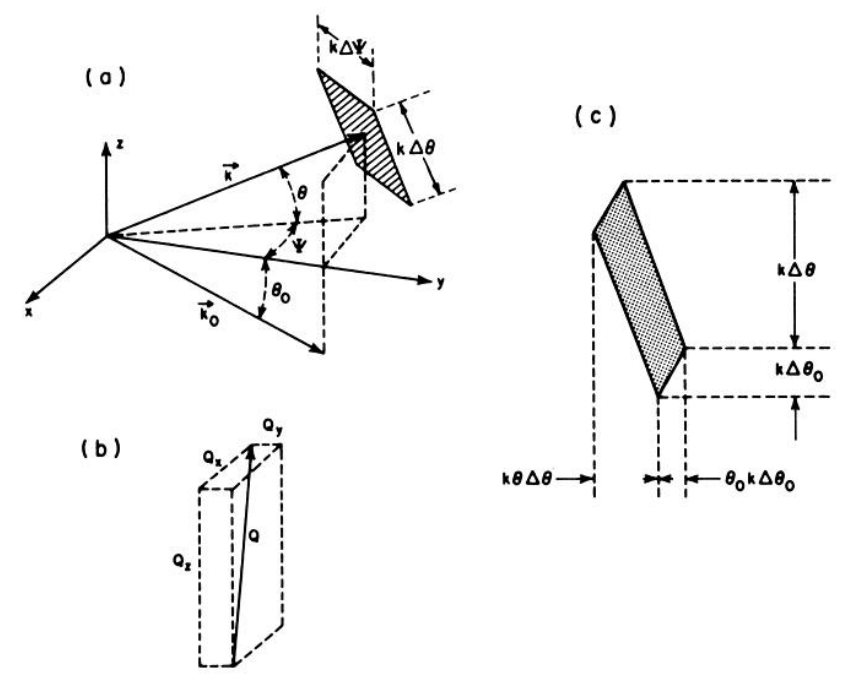

FIG. 3. Kinematics and resolution volume for a two-slit spectrometer. (a) Scattering kinematics, (b) wave-vector transfer, and (c) resolution volume. 
(a)

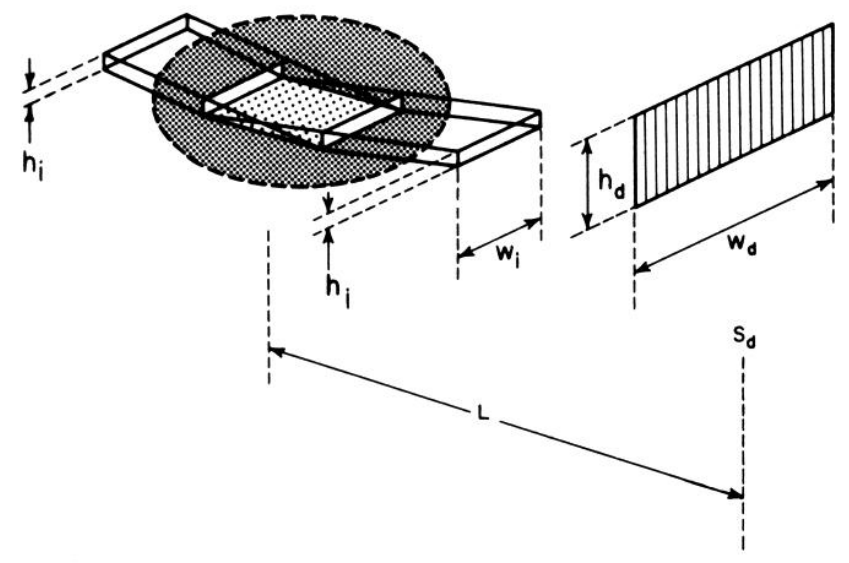

(b)

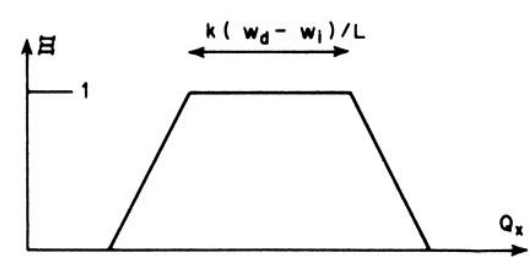

$\stackrel{k\left(w_{0}+w_{1}\right) / L}{\longrightarrow}$

FIG. 4. Spectrometer geometry and resolution cross section. (a) Two-slit experimental geometry. (b) Trapezoidal resolution function. 


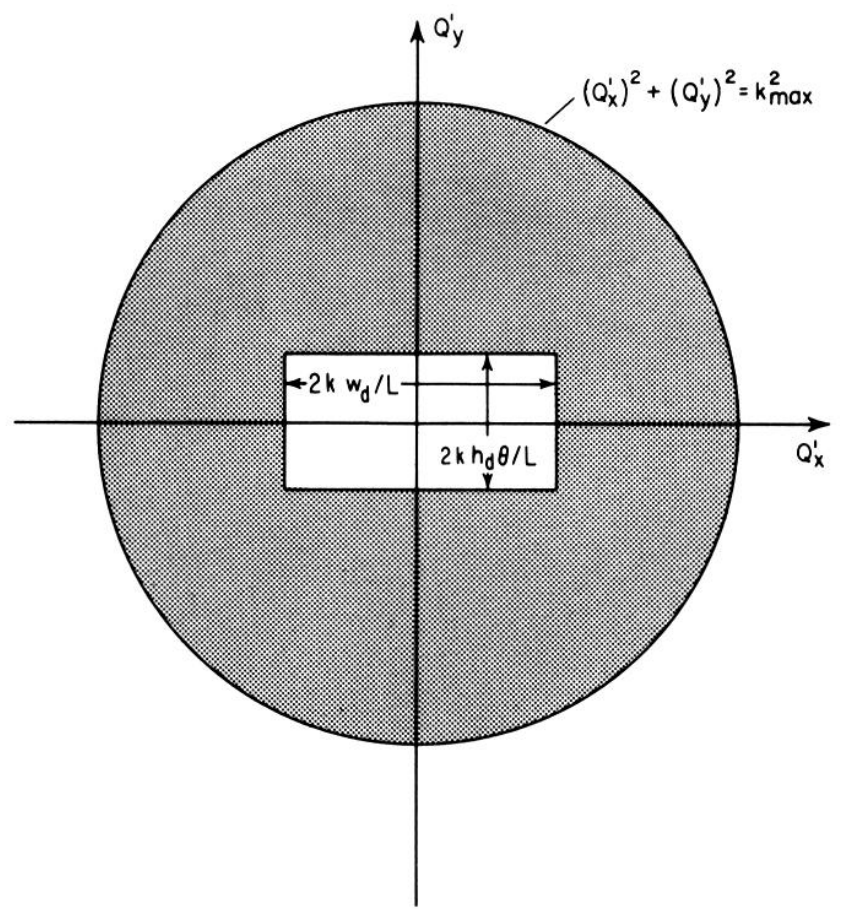

FIG. 5. Interception of the resolution volume with the $\mathbf{Q}_{x y}^{\prime}$ plane for a small incident beam slit and a large detector slit. The measured roughness arises from capillary-wave modes that scatter the reflected beam outside of the resolution volume of the detector. The integration in Eq. (46) is over the shaded area. 